\title{
CONSERVATION LAWS FOR EQUATIONS OF MIXED ELLIPTIC-HYPERBOLIC AND DEGENERATE TYPES
}

DANIELA LUPO and KEVIN R. PAYNE

\begin{abstract}
For partial differential equations of mixed elliptic-hyperbolic and degenerate types which are the Euler-Lagrange equations for an associated Lagrangian, invariance with respect to changes in independent and dependent variables is investigated, as are results in the classification of continuous one-parameter symmetry groups. For the variational and divergence symmetries, conservation laws are derived via the method of multipliers. The conservation laws resulting from anisotropic dilations are applied to prove uniqueness theorems for linear and nonlinear problems, and the invariance under dilations of the linear part is used to derive critical exponent phenomena and to obtain localized energy estimates for supercritical problems.
\end{abstract}

\section{Contents}

1. Introduction . . . . . . . . . . . . . . . . . . . . . 251

2. Symmetry groups . . . . . . . . . . . . . . . . . . . . . . 254

3. Associated conservation laws . . . . . . . . . . . . . . . . . . . . 262

4. Uniqueness theorems . . . . . . . . . . . . . . . . . . . . 266

5. Energy estimates for problems with supercritical growth . . . . . . . . . 273

6. Concluding remarks . . . . . . . . . . . . . . . . . . . . . . 281

A. Appendix . . . . . . . . . . . . . . . . . . . . . . . . 283

References . . . . . . . . . . . . . . . . . . . 288

\section{Introduction}

The association of conservation laws to the internal symmetries of a given partial differential equation that is the Euler-Lagrange equation associated to a Lagrangian is an old idea, going back to the work of E. Noether [23], which has been applied with

DUKE MATHEMATICAL JOURNAL

Vol. 127, No. 2, (C) 2005

Received 31 March 2003. Revision received 17 June 2004.

2000 Mathematics Subject Classification. Primary 35M10, 35L65, 58J70; Secondary 35A05, 35B33.

Authors' work supported by Ministero dell'Istruzione, dell'Universitá e della Ricerca project "Metodi Variazionali ed Equazioni Differenziali Non Lineari."

Payne's work supported by Ministero dell'Istruzione, dell’Universitá e della Ricerca project "Metodi Variazionali e Topologici nello Studio di Fenomeni Non Lineari." 
notable success in a wide range of primarily, but not exclusively, hyperbolic problems (cf. the Emmy Noether lecture given by Morawetz [22] at the International Congress of Mathematicians, Berlin, 1998). The associated conservation laws or, more generally, those associated to the linear part, when integrated have given rise to new energy estimates that in turn played a key role in results ranging from Morawetz's analysis (see [21]) of the decay in time of $L^{2}$-norms in space for solutions to the linear wave equation vanishing on the exterior of a starlike obstacle to Christodoulou and Klainerman's proof (see [5]) of the global stability of the Minkowski space in general relativity, as well as numerous results on decay, scattering, and regularity for nonlinear wave and Schrödinger equations. Moreover, invariance with respect to suitable scalings in nonlinear equations is well known to result in critical exponents related to concentration phenomena and the loss of compactness in both elliptic problems, as originated in the work of Brezis and Nirenberg [4], and hyperbolic problems, as is well summarized in the monograph of Strauss [31]. In addition, for two-dimensional problems, a conservation law locally gives rise to a potential function that under favorable circumstances can play a key role in uniqueness theorems, as was first shown by Morawetz [19] for a linear mixed-type problem.

The principal aim of this work is to bring these ideas to bear on equations of mixed elliptic-hyperbolic type in a systematic way by classifying the symmetries for an important class of model equations and then developing a basis for all of the associated conservation laws. This development is done in a global way by simultaneously treating the elliptic, hyperbolic, and type-changing regions and hence yields a typeindependent tool for the treatment of mixed and degenerate equations. More precisely, we study conservation laws associated to partial differential equations of the form

$$
K(y) \Delta_{x} u+\partial_{y}^{2} u+f(u)=0
$$

where $(x, y) \in \mathbf{R}^{N} \times \mathbf{R}, \Delta_{x}$ is the Laplace operator on $\mathbf{R}^{N}$ with $N \geq 1, f \in C^{0}(\mathbf{R})$, and the coefficient $K \in C^{0}(\mathbf{R})$ satisfies

$$
K(0)=0 \quad \text { and } \quad K(y) \neq 0 \quad \text { for } y \neq 0
$$

so that the equation degenerates along the hypersurface $y=0$. Our main interest is cases in which $K$ yields a change of type, that is, in which $K$ also satisfies

$$
y K(y)>0 \text { for } y \neq 0
$$

so that the equation (1.1) is of mixed type (elliptic for $y>0$ and hyperbolic for $y<0$ ), although much of what is discussed depends only on (1.1) and (1.2). We see that the richest possible structure for the symmetries results when $K$ takes a pure power form

$$
K(y)=y|y|^{m-1}, \quad m>0,
$$


in the mixed-type case or $\pm|y|^{m}$ in the purely elliptic/hyperbolic but degenerate cases. The linear part of equation (1.1) with (1.4) is known as the Gellerstedt equation, which was introduced in [11] for $N=1$ and which includes the Tricomi equation (see [34]) when $K(y)=y$, while the choice $K(y)=y^{2}$ yields the degenerate elliptic Grushin equation (see [14]). Equation (1.1) is the Euler-Lagrange equation for the Lagrangian

$$
\mathscr{L}_{f}(y, u, \nabla u)=\frac{1}{2}\left(K(y)\left|\nabla_{x} u\right|^{2}+u_{y}^{2}\right)+F(u)
$$

if $F$ is the primitive of $f$ which vanishes at the origin. In the linear homogeneous case, when $f=0$, we simply write $\mathscr{L}$ in place of $\mathscr{L}_{0}$; that is,

$$
\mathscr{L}(y, \nabla u)=\frac{1}{2}\left(K(y)\left|\nabla_{x} u\right|^{2}+u_{y}^{2}\right) .
$$

In fact, the class defined by (1.1) - (1.3) represents the simplest examples of secondorder equations of mixed type associated to a Lagrangian and hence is a natural class to investigate. Moreover, while the analysis can be done globally for this class, the resulting estimates can be localized near $y=0$, and hence equation (1.1) can be viewed as a family of canonical forms for mixed-type equations associated to a Lagrangian, where the parameter $m$ gives the order of degeneration.

Our main results are as follows. The classification of all continuous oneparameter symmetry groups for equation (1.1) with power-type degeneration (1.4) (or $K(y)= \pm|y|^{m}$ ) is given in Section 2 and culminates in Theorem 2.5, whose complete proof is given in the appendix. One finds, apart from certain trivial symmetries in the linear homogeneous case, symmetries that generate conservation laws coming from

(1) translations in the space variables $x$,

(2) rotations in the space variables,

(3) certain anisotropic dilations, and

(4) inversion with respect to a well-chosen hypersurface.

The second family is present only in space dimension $N \geq 2$, and the last two families are present only for the pure power degeneracies (1.4) and for associated critical pure power nonlinearities. The associated conservation laws are derived in Section 3 using the multiplier method. To the best of our knowledge, all of these conservation laws are new, with the exception of the translation invariance law in $N=1$, due to Morawetz [19], while the presence of the symmetries (1), (3), and (4) above were known for $N=1$ for the Tricomi equation (see [12]). The dilation invariance law is strongly related to certain Pohožaev type identities that give the first examples of critical exponent phenomena in a mixed elliptic-hyperbolic setting (see [18]) analogous to those known in the elliptic setting starting from the work of Pohožaev [27]. Applications of the dilation invariance laws are then given. In Section 4, we prove uniqueness theorems for linear and nonlinear boundary value problems of mixed and degenerate types 
in the plane $(N=1)$. In the linear case, we obtain uniqueness under less restrictive geometric assumptions on the hyperbolic boundary than those for known results (see the discussion at the beginning of Section 4). This improvement is of interest for applications to transonic flow (see Section 6 for details). In the nonlinear case, we obtain a uniqueness result that can be read as the nonexistence of nontrivial solutions for a problem at critical growth and hence serves to extend the analogy between the elliptic Dirichlet problem and boundary value problems for mixed and degenerate equations with respect to existence of nontrivial solutions and critical growth. Nonexistence is shown for mixed and degenerate problems with supercritical growth in [18], while existence is obtained for subcritical growth in [17], and the current result sets the stage for Brezis-Nirenberg-type results in which one would attempt to recover existence at critical growth by introducing a suitable perturbation of the nonlinearity. In Section 5 , we give some decay estimates for semilinear equations of degenerate hyperbolic type with supercritical growth. In particular, another critical exponent is derived by a dimensional analysis of the relevant energy functional and the concentration of the localized energy at a critical instant in time when the wave speed tends to zero is analyzed; the results so obtained extend to a degenerate hyperbolic situation the localized energy estimates of Struwe [33], Shatah and Struwe [29], and Grillakis [13]. Finally, some concluding remarks concerning physical and geometrical applications, as well as the underlying geometric structure of equations of the form (1.1), are given in Section 6.

\section{Symmetry groups}

In this section, we analyze the internal symmetries present in the mixed-type equation (1.1) in preparation for the derivation of the associated conservation laws. In order to render our treatment relatively self-contained, the basic notion and needed results from the theory of symmetry groups for differential equations is recalled using the book of Olver [24] as a constant point of reference.

\subsection{Background notions}

We begin by recalling that a group $G$ is a symmetry group for a differential equation

$$
\mathscr{F}\left(x, u, D u, \ldots, D^{k} u\right)=0, \quad k \in \mathbf{N},
$$

if one can define an action of $G$ on the space of smooth solutions of (2.1); that is, if $f(x)$ solves (2.1), then so does the transformed function $\tilde{f}=g \cdot f$ for each $g \in$ $G$. We consider only continuous (not discrete) transformation groups $G$ which act simultaneously on the dependent and independent variables. Such an action can be described in terms of the graph of the function $f$ (see [24, Section 2.2]), where, in general, the symmetry groups are local symmetry groups in the sense that a given group action may be meaningful only on some potentially small neighborhood of each 
given point in the domain of a differential equation or only for elements of the group near the identity. This local notion allows one to exploit a larger class of symmetries and results in infinitesimal techniques that can classify all such symmetries.

A stronger notion of a (local) symmetry group can be given for variational problems in which the differential equation (2.1) is the Euler-Lagrange equation associated to a Lagrangian. The linear part $L$ of (1.1) is of divergence form $L=\operatorname{div}\left(K(y) \nabla_{x}, \partial_{y}\right)$ with associated Lagrangian $\mathscr{L}_{f}$ given by (1.5) for which there is the following variational principle: Any sufficiently regular solution $u$ to equation (1.1) in $\Omega \subset \mathbf{R}^{N+1}$ is a stationary point of the functional

$$
J(u)=\int_{\Omega} \mathscr{L}_{f}(y, u, \nabla u) d x d y
$$

with respect to variations of $u$ of compact support, that is, with respect to variations $u_{\epsilon}=u+\epsilon \varphi$, where $\varphi \in C_{0}^{\infty}(\Omega)$ and $F$ is the primitive of $f \in C^{0}(\mathbf{R})$ which vanishes at the origin. A continuous and connected local group $G$ is said to be a variational symmetry group if the natural prolongation of the action of $G$ onto the Lagrangian leaves invariant the variational integral (2.2) (see [24, Section 4.2]). Concrete examples of variational symmetries are given in Section 2.2.

As a final preparatory remark, we recall that a given local symmetry group does not necessarily generate an associated conservation law; however, all variational symmetries do, as do the so-called divergence symmetries, whose definition is most easily given in infinitesimal terms. Such infinitesimal criteria can be used both to classify the symmetries and to construct the complete symmetry group under suitable general assumptions, as we do in Section 2.3.

\subsection{Explicit group representations}

First we consider invariance with respect to translations in $x \in \mathbf{R}^{N}$. In what follows, $\Omega$ is some arbitrary open set in $\mathbf{R}^{N}$ equipped with its standard basis $\left\{e_{k}: k=1, \ldots, N\right\}$ and norm $|\cdot|$. It is obvious that if $u \in C^{2}(\Omega)$ is a solution of equation (1.1), then for each $k=1, \ldots, N$,

$$
u_{k ; \epsilon}(x, y)=\mathscr{T}_{k ; \epsilon} u(x, y)=u\left(x-\epsilon e_{k}, y\right)
$$

is also a solution of (1.1) in the relevant translate of $\Omega$. Hence we have $N$ oneparameter symmetry groups of translations, which, by forming products of these generators, gives $\mathbf{R}^{N}$ as a symmetry group of translations in any $x$-direction. Moreover, each translation symmetry is a variational symmetry; that is, it preserves the functional $J$ of (2.2) in the following sense. Consider the simultaneous change of independent and dependent variables

$$
(x, y, u) \mapsto\left(x^{*}, y^{*}, u^{*}\right)=\left(x+\epsilon x_{0}, y, u\right)
$$


which gives the representation of the action of a general translation in the direction $x_{0} \in \mathbf{R}^{N}$ on the total space of dependent and independent variables. A routine calculation shows that we have

$$
J(u)=J\left(u^{*}\right)=\int_{\Omega^{*}}\left[\frac{1}{2}\left(K\left(y^{*}\right)\left|\nabla_{x^{*}} u^{*}\right|^{2}+\left(u_{y^{*}}^{*}\right)^{2}\right)+F\left(u^{*}\right)\right] d x^{*} d y^{*},
$$

where $\Omega^{*}$ is the $\epsilon$-translate of $\Omega$ in the direction $x_{0}$. It is worth pointing out that equations involving the mixed/degenerate-type operator $L$ do not possess an invariance with respect to translations in $y$ and that this deficiency creates substantial differences with respect to the strictly hyperbolic case of the wave operator, in which one has invariance with respect to translation by a timelike variable.

Next, we turn to rotations in $x \in \mathbf{R}^{N}$. For each pair of natural numbers $j, k=$ $1, \ldots, N$ with $N \geq 2$ and $j<k$, if $A_{j, k ; \epsilon}$ is the counterclockwise rotation by $\epsilon$ in the $x_{j} x_{k}$ plane and if $u \in C^{2}(\Omega)$ is a solution of equation (1.1), then so is

$$
u_{j, k ; \epsilon}(x, y)=\mathscr{R}_{j, k ; \epsilon} u(x, y)=u\left(A_{j, k ; \epsilon} x, y\right)
$$

in the rotated domain. Forming products of these generators, the orthogonal group $O(N)$ gives a variational symmetry group since one easily verifies (2.5) using the natural mapping

$$
(x, y, u) \mapsto\left(x^{*}, y^{*}, u^{*}\right)=\left(A^{-1}(\epsilon) x, y, u\right),
$$

where $A(\epsilon)$ is any smooth path in $O(N)$.

We now turn to anisotropic dilations. Here we specialize the type-change function $K(y)$, assuming that it has the pure power form (1.4). It is easy to verify that if $u \in$ $C^{2}(\Omega)$ is a solution of the linear homogeneous equation

$$
y|y|^{m-1} \Delta_{x} u+\partial_{y}^{2} u=0,
$$

then the scaled function

$$
u_{\lambda}(x, y)=\mathscr{S}_{\lambda} u(x, y)=\lambda^{-p(m, N)} u\left(\lambda^{-(m+2)} x, \lambda^{-2} y\right),
$$

where

$$
p(m, N)=\frac{N(m+2)-2}{2}>0,
$$

is also a solution of (2.8) for $\lambda>0$ and $(x, y)$ in a suitably scaled domain $\Omega^{*}$. Hence we have a multiplicative group $\mathbf{R}^{+}$of anisotropic dilations as a symmetry group for the linear homogeneous equation. This symmetry is again variational as the functional $J$ is invariant with respect to the natural mapping

$$
(x, y) \mapsto\left(x^{*}, y^{*}, u^{*}\right)=\left(\lambda^{m+2} x, \lambda^{2} y, \lambda^{-p(m, N)} u\right),
$$


where $p(m, N)$ is defined by (2.10). In general, a semilinear equation does not have this symmetry group of dilations, but for a certain critical power nonlinearity it is true, as we will see. We note that the choice of the scaling factor $\lambda^{-p(m, N)}$ is needed both to have variational symmetries and to allow for for a symmetry in the critical semilinear case.

Finally, we turn to inversions. We begin with the following observation, which we record for future reference.

PROPOSITION 2.1

Let $u \in C^{2}(\Omega)$ be a solution to the Gellerstedt equation (2.8). Then the inverted and scaled function

$$
v(x, y)=\Phi u(x, y)=|d|^{-q(N, m)} u\left(\frac{x}{d}, \frac{y}{d^{2 /(m+2)}}\right)
$$

is also a solution in $\Omega^{*}=\left\{(x, y):\left(d^{-1} x, d^{-2 /(m+2)} y\right) \in \Omega\right\} \backslash\{(x, y): d=0\}$, where

$$
q(m, N)=\frac{N(m+2)-2}{2(m+2)}>0
$$

and

$$
d(x, y)=|x|^{2}+\frac{4}{(m+2)^{2}} y|y|^{m+1} .
$$

A few comments about this result are in order. The zero level set $\Gamma_{0}=d^{-1}(0)$ of the function $d$ defined in (2.14) gives the backward characteristic cone for $L$ with vertex at the origin. The inversion is performed with respect to the hypersurface $\Gamma_{1}=$ $d^{-1}(1)$, which remains fixed, and the inversion preserves the orbits of the action of the anisotropic dilation group outside the solid backward light cone $\bar{\Sigma}_{0}=d^{-1}((-\infty, 0])$, as one sees from formula (2.12). Inside the cone $\Sigma_{0}$, the inversion in (2.12) does not preserve the orbits of this group; there is also a reflection with respect to the $y$-axis which is another symmetry in the equation. One could also extend the inversion (2.12) into $\Sigma_{0}$ in such a way as to preserve all of the orbits of the dilation group; namely,

$$
\tilde{v}(x, y)=\tilde{\Phi} u(x, y)=|d|^{-q(N, m)} u\left(\frac{x}{|d|}, \frac{y}{d^{2 /(m+2)}}\right) .
$$

The inversion (2.15) has the minor disadvantage that the multiplier $M u$ generated by (2.15) has a coefficient that is not $C^{1}$ for each $m>0$, while the multiplier (3.5) generated by (2.12) has $C^{1}$ coefficients. The factor $|d|^{-q(N, m)}$ is a singular solution of the Gellerstedt equation which, when cut off to $\Sigma_{0}$ or its complement, is proportional to a fundamental solution based at the origin, as is clearly explained in [2] for the case of the Tricomi equation. In two dimensions for the Tricomi operator (when $N=1$ and $m=1$ ), the level curves of $d$, with $d>0$, are the so-called normal curves 
of Tricomi [34], which played an important role in the first works on mixed-type equations. The inversions described here are known in this classical case (see [12]), but are written down only in special local coordinates in the elliptic and hyperbolic half-planes, respectively.

In order to extract a symmetry group from the invariance (2.12) (or (2.15)), two problems need to be addressed: the action is discrete and is not everywhere defined, even in the case $\Omega=\mathbf{R}^{N+1}$. (There is a singularity along $d^{-1}(0)$.) This also happens for inversion with respect to light cones for the wave equation and can be resolved by conjugating some other invariance with respect to the inversion; translations in $x$ provide the solution. What results are $N$ local transformation groups (one for each translation in $\mathbf{R}^{N}$ ), which is the content of the following proposition.

\section{PROPOSITION 2.2}

Let $u \in C^{2}(\Omega)$ be a solution of the Gellerstedt equation (2.8) in $\Omega$, and let $\left(x_{0}, y_{0}\right) \in$ $\Omega$ be fixed. Consider $k \in \mathbf{N}$ and $\epsilon \in \mathbf{R}$ with $1 \leq k \leq N$ and $|\epsilon|$ sufficiently small. Then the function

$$
u_{k ; \epsilon}(x, y)=\mathscr{I}_{k ; \epsilon} u(x, y)=D_{k, \epsilon}^{-q(N, m)} u\left(\frac{x+\epsilon d e_{k}}{D_{k, \epsilon}}, \frac{y}{D_{k, \epsilon}^{2 /(m+2)}}\right)
$$

gives a $C^{2}$-solution of (2.8) in a suitably transformed neighborhood $\Omega_{k, \epsilon}^{*}$ of $\left(x_{0}, y_{0}\right)$, where

$$
D_{k, \epsilon}(x, y)=1+2 \epsilon x_{k}+\epsilon^{2} d(x, y),
$$

$d(x, y)$ is defined by (2.14), and $q(N, m)$ is defined by (2.13).

We limit ourselves to a few comments about the proof. The inversion operators employed are nothing other than the composition

$$
\mathscr{I}_{k, \epsilon}=\Phi \circ \mathscr{T}_{k, \epsilon} \circ \Phi,
$$

where $\Phi$ is the inversion map of (2.12) and $\mathscr{T}_{k, \epsilon}$ is the translation (2.3). Being a composition of three transformations that carry one solution into another, the function defined by (2.16) is a solution if it is well defined. One needs only the fact that $D_{k, \epsilon}$ defined by (2.17) is positive and that $\left(D_{k, \epsilon}^{-1}\left(x+\epsilon d e_{k}\right), D_{k, \epsilon}^{-2 /(m+2)} y\right)$ lies in the domain $\Omega$, which happens locally for $|\epsilon|$ small enough. In addition, we remark that, as happens in the case of the wave operator, these local symmetry groups of inversions are not variational, as one can check by using the natural mapping

$$
(x, y, u) \mapsto\left(x^{*}, y^{*}, u^{*}\right)=\left(\frac{x-\epsilon d e_{k}}{D_{k,-\epsilon}}, \frac{y}{D_{k,-\epsilon}^{2 /(m+2)}}, D_{k, \epsilon}^{-q(N, m)} u\right) .
$$

However, they are divergence symmetries, as is shown below. 


\subsection{Classifying the symmetries}

We conclude Section 2 by addressing the question of whether the (local) oneparameter symmetry groups presented above generate the complete symmetry group for the linear homogeneous equation associated to (1.1). We begin by noting that each invariance previously considered is a combination of coordinate changes and scalings; sufficient conditions for having an invariance in the linear homogeneous case can be written as a nonlinear system of partial differential equations. We record this fact, which can be used to prove Propositions 2.1 and 2.2.

\section{PROPOSITION 2.3}

If $u$ is a solution to $K(Y) \Delta_{Y} u+\partial_{Y}^{2} u=0$ in a neighborhood $\Omega$ of $\left(X_{0}, Y_{0}\right)=$ $\left(X\left(x_{0}, y_{0}\right), Y\left(x_{0}, y_{0}\right)\right) \in \mathbf{R}^{N+1}$, then $v(x, y)=\psi(x, y) u(X(x, y), Y(x, y))$ is also a solution in a neighborhood $\Omega^{*}$ of $\left(x_{0}, y_{0}\right)$, provided that the functions $\psi, X=$ $\left(X_{1}, \ldots, X_{N}\right), Y$ are $C^{2}\left(\Omega^{*}\right)$ and satisfy the system; for $j, k=1, \ldots, N, j \neq k$,

$$
\begin{gathered}
L \psi=L X_{j}=L Y=0, \\
\left\langle\nabla \psi, \nabla X_{j}\right\rangle_{K}=\langle\nabla \psi, \nabla Y\rangle_{K}=\left\langle\nabla X_{j}, \nabla Y\right\rangle_{K}=\left\langle\nabla X_{j}, \nabla X_{k}\right\rangle_{K}=0, \\
\left\langle\nabla X_{j}, \nabla X_{j}\right\rangle_{K}=(K \circ Y)\langle\nabla Y, \nabla Y\rangle_{K},
\end{gathered}
$$

where $L=K(y) \Delta_{x}+\partial_{y}^{2}, K(y)$ satisfies (1.2), and $\langle\nabla v, \nabla w\rangle_{K}:=v_{y} w_{y}+K(y) \nabla_{x} v$. $\nabla_{x} w$.

If one is able to construct, at least for $|\epsilon|$ small, a smooth one-parameter family $X(x, y ; \epsilon), Y(x, y ; \epsilon), \psi(x, y ; \epsilon)$ of such transformations, as is done above, then one has sufficient conditions for the generator of a (local) one-parameter symmetry group. On the other hand, necessary and sufficient conditions can be obtained that theoretically allow for a complete calculation of the symmetry group via infinitesimal criteria. Such criteria require nondegeneracy hypotheses on the differential equation which are satisfied by the linear Gellerstedt equation (cf. formulas (A.2), (A.3)), as well as regularity in the coefficients. Hence, in order to apply directly this machinery in a global way, we consider the second-order linear homogeneous equation with smooth coefficients

$$
L u=y^{m} \sum_{j=1}^{N} u_{x_{j} x_{j}}+u_{y y}=0, \quad m \in \mathbf{N}=\{1,2, \ldots\},
$$

which is the mixed-type Gellerstedt equation with $m \in N$ odd and the degenerate elliptic equation of Grushin type for $m \in \mathbf{N}$ even. However, the analysis shows that the resulting classification remains valid in general for $K(y)= \pm|y|^{m}$ or $y|y|^{m-1}$ with $m>0$, as is noted below. The infinitesimal criteria for symmetry groups associated 
to equation (2.19) are expressed in terms of the generating vector fields

$$
v=\sum_{i=1}^{N} \xi^{i}(x, y, u) \frac{\partial}{\partial x_{i}}+\eta(x, y, u) \frac{\partial}{\partial y}+\varphi(x, y, u) \frac{\partial}{\partial u},
$$

which act on an open subset $M$ of the 0 -jet space $\mathbf{R}^{N+1} \times \mathscr{U}^{(0)} \simeq \mathbf{R}^{N+1} \times \mathbf{R}$ (the space of values for independent and dependent variables) together with the action of their prolongations onto higher-order jet spaces (which includes the values of higherorder derivatives of $u$ ). In particular, one has the following general result, which we state as a lemma in our situation (cf. [24, Theorems 2.71, 4.12, 4.34])

\section{LEMMA 2.4}

Consider the nondegenerate differential equation (2.19) of second order and a smooth vector field $v$ given by (2.20). Then

(a) $\quad v$ generates a local one-parameter group of symmetries for (2.19) if and only if

$$
\operatorname{pr}^{(2)} v\left[y^{m} \sum_{j=1}^{N} u_{x_{j} x_{j}}+u_{y y}\right]=0
$$

for every solution $u$ of (2.19), where $\mathrm{pr}^{(2)} v$ is the second prolongation of $v$ onto the 2-jet space $\mathbf{R}^{N+1} \times \mathscr{U}^{(2)}$;

(b) $v$ generates a one-parameter group of variational symmetries for (2.19) if and only if

$$
\operatorname{pr}^{(1)} v\left[\mathscr{L}\left(y, u_{x_{1}}, \ldots, u_{x_{N}}, u_{y}\right)\right]+\mathscr{L}\left(y, u_{x_{1}}, \ldots, u_{x_{N}}, u_{y}\right) \operatorname{Div} \Xi=0,
$$

where $\mathrm{pr}^{(1)} v$ is the first prolongation of $v$ onto the 1-jet space $\mathbf{R}^{N+1} \times \mathscr{U}^{(1)}$, $\mathscr{L}\left(y, u_{x_{1}}, \ldots, u_{x_{N}}, u_{y}\right)=\left(y^{m} \sum_{j=1}^{N} u_{x_{j}}^{2}+u_{y}^{2}\right) / 2$ is the Lagrangian associated to (2.19), and Div is the total divergence operator that acts via the chain rule on the vector-valued function $\Xi=\left(\xi^{1}, \ldots, \xi^{N}, \eta\right)$.

A vector field $v$ which satisfies (2.22) is called an infinitesimal variational symmetry of $\mathscr{L}$, and since it generates a variational symmetry for the associated Euler-Lagrange equation, it produces a conservation law for the solutions of (2.19) by Noether's theorem (cf. [24, Theorem 4.29]). A conservation law for (2.19) also results from the presence of an infinitesimal divergence symmetry that is a vector field $v$ such that there exists a vector-valued function $B=B(x, y, u)$ on the 0 -jet space $X \times \mathscr{U}^{(0)}$ such that

$$
\operatorname{pr}^{(1)} v\left[\mathscr{L}\left(y, u_{x_{1}}, \ldots, u_{x_{N}}, u_{y}\right)\right]+\mathscr{L}\left(y, u_{x_{1}}, \ldots, u_{x_{N}}, u_{y}\right) \operatorname{Div} \Xi=\operatorname{Div} B,
$$

where again Div is the total divergence operator (cf. [24, Section 4.4]). 
The infinitesimal generators of the group actions by translation, dilation, rotation, and inversion considered above are computed via formulas (2.4), (2.9), (2.11), and (2.18) and yield

$$
\begin{aligned}
v_{k}^{T}= & \frac{\partial}{\partial x_{k}}, \quad k=1, \ldots, N, \\
v^{D}= & (m+2) x \cdot \nabla_{x}+2 y \frac{\partial}{\partial y}-\frac{N(m+2)-2}{2} u \frac{\partial}{\partial u}, \\
v_{j k}^{R}= & x_{k} \frac{\partial}{\partial x_{j}}-x_{j} \frac{\partial}{\partial x_{k}}, \quad 1 \leq j<k \leq N, \\
v_{k}^{I}= & -d(x, y) \frac{\partial}{\partial x_{k}}+2 x_{k} x \cdot \nabla_{x}+\frac{4}{m+2} x_{k} y \frac{\partial}{\partial y} \\
& -\frac{N(m+2)-2}{m+2} x_{k} u \frac{\partial}{\partial u}, \quad k=1, \ldots, N .
\end{aligned}
$$

These vector fields can be shown to generate almost all of the symmetry group for (2.19). The rest of the group comes from linearity and homogeneity; in particular, if $u$ solves (2.19) in $\Omega$, then for every $\epsilon \in \mathbf{R}$ the functions $(1+\epsilon) u$ and $u+\epsilon \beta$ are also solutions for any solution $\beta$ of (2.19). The infinitesimal generators of this trivial part of the symmetry group are

$$
v^{u}=u \frac{\partial}{\partial u} \quad \text { and } \quad v^{\beta}=\beta \frac{\partial}{\partial u}, \quad L \beta=0 .
$$

One has the following classification of the symmetries.

\section{THEOREM 2.5}

For each open subset $M$ of the 0 -jet $\mathbf{R}^{N+1} \times \mathbf{R}$, consider the set $\mathscr{V}(M)$ of vector fields $v$ with smooth coefficients in $M$ as given by (2.20).

(a) The set of infinitesimal generators $v$ of a one-parameter symmetry group for equation (2.19) form an infinite-dimensional Lie subalgebra $\mathscr{A}(M)$ of $\mathscr{V}(M)$ with basis

$$
\left\{v^{u}, v^{\beta}, v_{k}^{T}, v^{D}, v_{j k}^{R}, v_{k}^{I}: j, k=1, \ldots, N, k>j\right\},
$$

where $\beta$ is any solution of $L \beta=0$ and the vector fields are defined by (2.24)(2.28).

(b) The set of infinitesimal generators $v$ of a one-parameter variational symmetry group for equation (2.19) form an $(N+2+N(N-1) / 2)$-dimensional Lie subalgebra of $\mathscr{A}(M)$ with basis given by $\left\{v^{\beta}, v_{k}^{T}, v^{D}, v_{j k}^{R}: j, k=1, \ldots, N, k>\right.$ $j\}$, where $\beta$ is any fixed constant.

(c) The set of infinitesimal generators $v$ of a one-parameter group of divergence symmetries for equation (2.19) form an infinite-dimensional vector subspace 
of $\mathscr{V}(M)$ with basis given by $\left\{v^{\beta}, v_{k}^{T}, v^{D}, v_{j k}^{R}, v_{k}^{I}: j, k=1, \ldots, N, k>j\right\}$, where $\beta$ is any solution of $L \beta=0$.

The proof of Theorem 2.5 essentially amounts to a sequence of lengthy but elementary calculations which, for completeness, are given in the appendix. Here we make only a few comments. The theorem effectively calculates the connected component of the identity of the full symmetry group for equation (2.19) since it is enough to exponentiate the generators to arrive at the corresponding connected local Lie group (cf. [24, Section 2.4, Corollary 2.40]). The coefficients of the generators are linear in $u$, and hence the neighborhood $M$ in the 0 -jet can be chosen as $\Omega \times \mathbf{R}$, which also follows from the explicit representations of the group actions given above. Moreover, the classification continues to hold for $m \in \mathbf{R}^{+} \backslash \mathbf{N}$ when the coefficients are not smooth as one can first apply the classification in the open components of $\mathbf{R}^{N+1} \backslash\{y=0\}$ to obtain the symmetry groups over the components which have a continuous extension across the interface $\{y=0\}$ using the representations of Section 2.2. Finally, we note that, as in the case of the Laplace and wave equations, the symmetry group is strongly related to the conformal transformations with respect to an underlying singular geometric structure (see Section 6 for details).

\section{Associated conservation laws}

Having classified the (local) variational and divergence symmetry groups for our mixed-type equations, we are now ready to derive the conservation laws that are generated by the symmetries. By a conservation law associated to a given second-order equation of the form $L u+f(u)=0$ with independent variables $(x, y)$ we mean a first-order equation in divergence form $\operatorname{div}(U)=0$ which must be satisfied by every sufficiently regular solution $u$ of the given equation. Here $U=U(x, y, u, \nabla u, f)$ is some vector field whose dependence on $u$ is generally strongly nonlinear. While Noether's theorem (or its generalization) ensures the presence of a conservation law for each variational or divergence symmetry, and while there are also explicit formulas for the resulting conservation laws, we prefer to derive the conservation laws via the method of multipliers. This method is a differential version of the so-called $a b c$ integral method of Friedrichs as first used by Protter [28] and developed into a general theory in [8] for obtaining energy inequalities. One multiplies the given equation by a first-order differential expression of the form

$$
M u=\sum_{j=1}^{N} a_{j}(x, y) u_{x_{j}}+b(x, y) u_{y}+c(x, y) u
$$

and seeks to rewrite the equation $M u(L u+f(u))=0$ as the divergence of some $U$, where the coefficients $\left(a_{1}, \ldots, a_{N}, b, c\right)$ of the multiplier $M u$ are to be determined. 
In our case, since $L=K(y) \Delta_{x}+\partial_{y}^{2}$ is associated to a Lagrangian, a conservation law results by picking $M u$ as the first variation at the origin of a one-parameter family of transformed solutions under the action of a variational or divergence symmetry group (cf. [31, Section 2]). In fact, it suffices to pick $M u$ associated to the linear part, and subsequently one determines the admissible nonlinearities. The classification result Theorem 2.5 shows that $M u$ must have the form (3.1) for the nontrivial symmetries and gives the complete family of multipliers which are natural from an algebraic-geometric point of view for use in the $a b c$ integral method. Our preference for the multiplier method is due, in part, to the fact that even if a resulting combination does not yield a conservation law, it may be useful nonetheless, and all of the basic identities are clearly displayed as building blocks.

The first variations of the one-parameter families of transformed solutions under the action of the one-parameter groups of translation, rotation, dilation, and inversion are

$$
\begin{aligned}
M u= & \left.\frac{d}{d \epsilon} \mathscr{T}_{k ; \epsilon} u\right|_{\epsilon=0}=-u_{x_{k}}, \\
M u= & \left.\frac{d}{d \epsilon} \mathscr{R}_{j, k ; \epsilon} u\right|_{\epsilon=0}=x_{j} u_{x_{k}}-x_{k} u_{x_{j}}, \\
M u= & \left.\frac{d}{d \lambda} \mathscr{S}_{\lambda} u\right|_{\lambda=1}=-(m+2) x \cdot \nabla_{x} u-2 y u_{y}-\frac{N(m+2)-2}{2} u, \\
M u= & \left.\frac{d}{d \epsilon} \mathscr{I}_{k, \epsilon} u\right|_{\epsilon=0}=d(x, y) u_{x_{k}}-2 x_{k} x \cdot \nabla_{x} u-\frac{4}{m+2} x_{k} y u_{y} \\
& -\frac{N(m+2)-2}{m+2} x_{k} u,
\end{aligned}
$$

where $d(x, y)=|x|^{2}+4 y|y|^{m+1} /(m+2)^{2}$ is as introduced in (2.14) and we have used formulas (2.3), (2.6), (2.9), and (2.16). In addition, there are the trivial variational and divergence symmetries for the linear homogeneous equation whose one-parameter family of solutions is $u+\epsilon \beta$ with $\beta$ any solution of the equation. The first variation in $\epsilon=0$ is $\beta$ and hence is not of the form (3.1), but the choices of $\beta$ constant and nonconstant give rise to the trivial conservation laws

$$
\begin{aligned}
\operatorname{div}\left(K(y) \nabla_{x} u, u_{y}\right) & =0, \\
\operatorname{div}\left(K(y)\left(\beta \nabla_{x} u-u \nabla_{x} \beta\right), \beta u_{y}-u \beta_{y}\right) & =0 .
\end{aligned}
$$

The first reflects the fact that the equation itself is in divergence form, and the second gives a so-called reciprocity relation but is of little interest for our purposes since it carries none of the particular structure of the equation.

Before stating the nontrivial conservation laws, we note that the space of multipliers to be used has as its generators $u, u_{y}, u_{x_{j}}$, which, when multiplying the equation 
$L u+F^{\prime}(u)=0$, yield the identities

$$
\begin{gathered}
\operatorname{div}\left(K(y) u \nabla_{x} u, u u_{y}\right)=u_{y}^{2}+K(y)\left|\nabla_{x} u\right|^{2}-u F^{\prime}(u), \\
\operatorname{div}\left(K(y) u_{y} \nabla_{x} u, \frac{1}{2}\left(u_{y}^{2}-K(y)\left|\nabla_{x} u\right|^{2}\right)+F(u)\right)=-\frac{1}{2} K^{\prime}(y)\left|\nabla_{x} u\right|^{2}, \\
(F(u)-\mathscr{L}(y, \nabla u))_{x_{j}}+\operatorname{div}\left[u_{x_{j}}\left(K(y) \nabla_{x} u, u_{y}\right)\right]=0,
\end{gathered}
$$

respectively, where $\mathscr{L}(y, \nabla u)$ is defined by (1.6). From (3.7), one sees that there is no conservation law in such equations associated to translations in $y$ (one would need $K$ constant), and from (3.8) one has the conservation law coming from translations in $x_{j}$. For our purposes the following commutator identities are also useful; those whose multipliers are $y u_{y}$ and $x_{j} u_{x_{j}}$, respectively, yield

$$
\begin{aligned}
\operatorname{div}\left(y K(y) u_{y} \nabla_{x} u, y(\right. & \left.\left.\frac{1}{2}\left(u_{y}^{2}-K(y)\left|\nabla_{x} u\right|^{2}\right)+F(u)\right)\right) \\
& =\frac{1}{2}\left(u_{y}^{2}-K(y)\left|\nabla_{x} u\right|^{2}\right)+F(u)-\frac{y}{2} K^{\prime}(y)\left|\nabla_{x} u\right|^{2}
\end{aligned}
$$

and

$$
\begin{aligned}
{\left[x_{j}(F(u)-\mathscr{L}(y, \nabla u))\right]_{x_{j}}+\operatorname{div}\left[x_{j} u_{x_{j}}\right.} & \left.\left(K(y) \nabla_{x} u, u_{y}\right)\right] \\
& =K(y) u_{x_{j}}^{2}-\mathscr{L}(y, \nabla u)+F(u) .
\end{aligned}
$$

We are now ready to give the main results of this section. We assume from here on that $u$ is a sufficiently smooth solution of the equation $K(y) \Delta_{x} u+\partial_{y}^{2} u+f(u)=0$ in some open set $\Omega \subset \mathbf{R}^{N} \times \mathbf{R}$. Fixing an element $a \in \mathbf{R}^{N}$, using $M u=a \cdot \nabla u$ as a multiplier which generates the translation in the direction $a$, and exploiting identity (3.8), we find that $u$ must satisfy the translation identity

$$
\operatorname{div}\left((F(u)-\mathscr{L}(y, \nabla u)) a+\left(a \cdot \nabla_{x} u\right) K(y) \nabla_{x} u,\left(a \cdot \nabla_{x} u\right) u_{y}\right)=0 .
$$

This identity is a conservation law that in the purely hyperbolic case is interpreted as a conservation of linear momentum and is valid for any space dimension, for each type-change function $K$, and for each nonlinearity $f$. Another family of conservation laws comes from the invariance with respect to the spatial rotations generated by the multipliers (3.3), which yield the rotation identity (where $j \neq k=1, \ldots, N$ )

$$
\begin{aligned}
\operatorname{div}[ & \left(x_{j} u_{x_{k}}-x_{k} u_{x_{j}}\right) K(y) \nabla_{x} u \\
& \left.+(F-\mathscr{L}(y, \nabla u))\left(x_{j} e_{k}-x_{k} e_{j}\right),\left(x_{j} u_{x_{k}}-x_{k} u_{x_{j}}\right) u_{y}\right]=0 .
\end{aligned}
$$

These identities are conservation laws, again valid for each $K$ and $f$, and can be interpreted as a conservation of angular momentum. 
In order to implement symmetries of dilation and inversion type, we now specialize to the case of the semilinear Gellerstedt equation. By using the multiplier (3.4), which generates the invariance under anisotropic dilations for the homogeneous equation, a suitable linear combination of the identities (3.10), (3.9), and (3.6) yields the dilation identity

$$
\begin{aligned}
& \operatorname{div}\left((m+2)(F(u)-\mathscr{L}(y, \nabla u)) x-y|y|^{m-1} M u \nabla_{x} u, 2 y(F(u)-\mathscr{L}(y, \nabla u))\right. \\
& \left.-u_{y} M u\right)=(N(m+2)+2) F(u)-\frac{N(m+2)-2}{2} u F^{\prime}(u),
\end{aligned}
$$

where $M u$ is given by (3.4) and $\mathscr{L}(y, \nabla u)$ is defined in (1.6). One sees that this identity is a conservation law if and only if the nonlinearity $f$ satisfies

$$
(N(m+2)+2) F(u)-\frac{N(m+2)-2}{2} u F^{\prime}(u)=0 .
$$

This happens in particular for the linear homogeneous equation $(f=0)$, as well as for power-type nonlinearities $f(u)=C u|u|^{p-2}$ with $C \in \mathbf{R}$, in the critical case

$$
p=2^{*}(N, m)=\frac{2(N(m+2)+2)}{N(m+2)-2} .
$$

This critical exponent $2^{*}(N, m)$ is the classical Sobolev exponent $2^{*}(d)=2 d /(d-2)$, where $d=1+N(m+2) / 2$ is the so-called homogeneous dimension of $\mathbf{R}^{N+1}$. That is, while the topological dimension of the space of independent variables $\mathbf{R}^{N+1}$ is of course $N+1$, associated to the anisotropic scaling invariance for the degenerate operator $L$ there is a natural non-Euclidian metric (see [6]) whose metric balls of radius $r$ have Euclidian volume that grows like $r^{d}$. It is this homogeneous dimension $d$ and not the topological dimension $N+1$ that controls the critical exponent phenomena, as has been observed in the analysis on certain Lie groups (see [10]) from which the term homogeneous dimension has been borrowed. The critical exponent (3.14) has been discussed by the authors in the case $N=1$ (see [18]).

Finally, by using the multiplier (3.5), which generates the $k$ th invariance under inversions in the homogeneous equation, we find the inversion identities (where $k=$ $1, \ldots, N)$

$$
\begin{aligned}
& \operatorname{div}\left(\left(2 d(x, y)(F(u)-\mathscr{L}(y, \nabla u))+\beta K(y) u^{2}\right) e_{k}-4 x_{k}(F(u)-\mathscr{L}(y, \nabla u)) x\right. \\
& \quad+\left(2 d(x, y) u_{x_{k}}-4 x_{k}\left(x \cdot \nabla_{x} u\right)-\frac{8 x_{k}}{m+2} y u_{y}-2 \beta x_{k} u\right) K(y) \nabla_{x} u \\
& 2 d(x, y) u_{x_{k}} u_{y}-4 x_{k}\left(x \cdot \nabla_{x} u\right) u_{y}-\frac{4 x_{k} y}{m+2}\left(u_{y}^{2}-K(y)\left|\nabla_{x} u\right|^{2}+2 F(u)\right) \\
& \left.\quad-2 \beta x_{k} u u_{y}\right)=2 x_{k}\left(\beta u F^{\prime}(u)-\left(2 N+\frac{4}{m+2}\right) F(u)\right)
\end{aligned}
$$


where $K(y), d(x, y)$, and $\mathscr{L}(y, \nabla u)$ are given by (1.4), (2.14), and (1.6), respectively, and $\beta=(N(m+2)-2) /(m+2)$ is the coefficient of $u$ in the inversion multiplier (3.5). These identities give conservation laws in both the linear homogeneous and critical semilinear cases with the same critical exponent as in the dilation case.

For future reference and to summarize the above discussion, we now give a few examples.

Example 3.1 (Linear Tricomi equation in the plane)

Let $u \in C^{2}(\Omega)$ solve the equation $u_{y y}+y u_{x x}=0$ in an open subset $\Omega$ of $\mathbf{R}^{2}$. Then one has conservation laws of translation, dilation, and inversion type; namely,

$$
\begin{aligned}
& \operatorname{div}\left(y u_{x}^{2}-u_{y}^{2}, 2 u_{x} u_{y}\right)=0 \\
& \operatorname{div}\left(y u u_{x}+3 x y u_{x}^{2}+4 y^{2} u_{x} u_{y}-3 x u_{y}^{2}, u u_{y}-2 y^{2} u_{x}^{2}+6 x u_{x} u_{y}+2 y u_{y}^{2}\right)=0 \\
& \operatorname{div}\left(\left(4 y^{3}-9 x^{2}\right)\left(y u_{x}^{2}-u_{y}^{2}\right)-24 x y^{2} u_{x} u_{y}-6 x y u u_{x}+3 y u^{2}\right. \\
& \left.\quad 2\left(4 y^{3}-9 x^{2}\right) u_{x} u_{y}-12 x y\left(u_{y}^{2}-y u_{x}^{2}\right)-6 x u u_{y}\right)=0
\end{aligned}
$$

Example 3.2 (Semilinear Gellerstedt equations with critical exponent in the plane)

Let $u \in C^{2}(\Omega)$ solve the equation $u_{y y}+K(y) u_{x x}+f(u)=0$ in an open subset $\Omega$ of $\mathbf{R}^{2}$, where $K(y)=y|y|^{m-1}$ and $f(u)=C u|u|^{p-2}$ with $p=2^{*}(1, m)=$ $2(m+4) / m$ and primitive $F(u)=C|u|^{p} / p$. Then one has conservation laws of translation, dilation, and inversion type; namely,

$$
\begin{aligned}
& \operatorname{div}\left(K(y) u_{x}^{2}-u_{y}^{2}+2 F(u), 2 u_{x} u_{y}\right)=0, \\
& \operatorname{div}\left(m K(y) u u_{x}+(m+2) x K(y) u_{x}^{2}+4 y K(y) u_{x} u_{y}-(m+2) x u_{y}^{2}\right. \\
& \quad+2(m+2) x F(u), m u u_{y}-2 y K(y) u_{x}^{2}+2(m+2) x u_{x} u_{y} \\
& \left.\quad+2 y u_{y}^{2}+4 y F(u)\right)=0, \\
& \quad \operatorname{div}\left(\left(4 y^{2} K(y)-(m+2)^{2} x^{2}\right)\left(K(y) u_{x}^{2}-u_{y}^{2}+2 F(u)\right)-8(m+2) x y K(y) u_{x} u_{y}\right. \\
& \quad-2 m(m+2) x K(y) u u_{x}+m(m+2) K(y) u^{2}, 2\left(4 y^{2} K(y)-(m+2)^{2} x^{2}\right) u_{x} u_{y} \\
& \left.\quad-4(m+2) x y\left(u_{y}^{2}-K(y) u_{x}^{2}+2 F(u)\right)-2 m(m+2) x u u_{y}\right)=0 .
\end{aligned}
$$

\section{Uniqueness theorems}

In this section, we consider the problem of uniqueness for sufficiently regular solutions to boundary value problems in the plane for linear and semilinear equations of mixed or degenerate type. It is well known that the question of what constitutes a well-posed problem for an equation of mixed type is a very delicate matter in which known results involve technical restrictions on the boundary geometry. Typically, data is placed only on a proper subset $\Gamma$ of the hyperbolic boundary, and, in known results, 
each component of $\Gamma$ is assumed to be monotone. We show that for Gellerstedt equations, monotonicity of $\Gamma$ can be weakened to a suitable starlike condition. Inspired by Morawetz's use of translation invariance for a linear mixed-type equation, we exploit the dilation potential, whose existence is guaranteed by the conservation law generated by anisotropic dilations. In the linear case, our results are complementary to hers. On one hand, the monotonicity needed of her translation potential requires $\Gamma$ to be monotone, while, on the other hand, our dilation potential exists only for a more restricted class of equations. Moreover, while the dilation potential remains monotone on starlike boundaries, it loses pointwise monotonicity on interior characteristic segments, but this difficulty is overcome by the use of a sharp Hardy-Sobolev inequality introduced in [18].

More precisely, we consider the equation

$$
L u=u_{y y}+y|y|^{m-1} u_{x x}=f
$$

with $m>0$ and $f=f(x, y)$ or $f=f(u)=C u|u|^{p-2}$ with $p=2^{*}(1, m)=2(m+$ 4) $/ m$. We consider (4.1) in an open and bounded set $\Omega \subset \mathbf{R}^{2}$ with piecewise $C^{1}$ boundary $\partial \Omega$ such that $\Omega$ is star-shaped with respect to the flow of the vector field $D=$ $-(m+2) x \partial_{x}-2 y \partial_{y}$ which generates the anisotropic dilation invariance. This means that for each $\left(x_{0}, y_{0}\right) \in \bar{\Omega}$, one has $\mathscr{F}_{t}\left(x_{0}, y_{0}\right) \in \bar{\Omega}$ for each $t \in[0,+\infty]$, where $\mathscr{F}_{t}\left(x_{0}, y_{0}\right)=\left(x_{0} e^{-(m+2) t}, y_{0} e^{-2 t}\right)$ is the time- $t$ flow of $\left(x_{0}, y_{0}\right)$ along $D$ (cf. [18, Definition 2.1]). We recall that $\Omega$ is simply connected and has a $D$-starlike boundary in the sense that $((m+2) x, 2 y) \cdot v(x, y) \geq 0$ for each regular point $(x, y) \in \partial \Omega$, where $v$ is the exterior unit normal (cf. [18, Lemma 2.2]). Without loss of generality, we may assume that the origin is a boundary point of $\Omega$ by exploiting the invariance with respect to translations in $x \in \mathbf{R}$.

Our first uniqueness result is for the degenerate hyperbolic Goursat problem in which the domain $\Omega^{-}$is a curvilinear triangle in the hyperbolic region, where $y<0$, with boundary $A B \cup B C \cup \Gamma_{1}$, where $A B$ is the parabolic segment $\left\{(x, 0) \in \mathbf{R}^{2}: x_{0} \leq\right.$ $x \leq 0\}, B C$ is the characteristic of $L$ with positive slope which joins $B=(0,0)$ to some point $C=\left(x_{C}, y_{C}\right)$ with $x_{C}, y_{C}<0$, and $\Gamma_{1}$ is a piecewise $C^{1}$-graph $y=y(x)$ joining $A=\left(x_{0}, 0\right)$ to $C$ which is assumed to be subcharacteristic (for $L$ ) (i.e., one assumes $1+y|y|^{m-1}(d y / d x)^{2} \geq 0$ along $\left.\Gamma_{1}\right)$. We call such a domain a Goursat domain. We consider classical solutions in $\Omega$, which is to say, $u \in C^{2}(\Omega) \cap C^{1}(\bar{\Omega} \backslash$ $\{A, B\}) \cap C^{0}(\bar{\Omega})$, which is the optimal regularity that one expects for such mixed-type problems and enough regularity to ensure the validity of a maximum principle in the case $\Gamma_{1}$ characteristic (see [1]).

THEOREM 4.1

Let $\Omega^{-}$be a Goursat domain that is star-shaped with respect to the vector field $D=$ 
$-(m+2) x \partial_{x}-2 y \partial_{y}$ with $\Gamma_{1}$ subcharacteristic. Let $u$ be a classical solution of the Goursat problem: $u$ satisfies (4.1) in $\Omega^{-}$with $u=0$ on $A B \cup \Gamma_{1}$. Then $u$ is unique.

\section{Proof}

The proof is given in 5 steps.

Step 1 (Introduce the dilation potential). Since the difference $u$ of any two solutions satisfies $L u=0$ in $\Omega^{-}$, one has the conservation $\operatorname{law} \operatorname{div}(U)=0$, where the vector field $U=\left(U_{1}, U_{2}\right)$ is given by substituting $F=0$ into (3.20). Since $\Omega^{-}$is simply connected, the conservative vector field $V=\left(V_{1}, V_{2}\right)=\left(U_{2},-U_{1}\right)$ admits a potential function $\varphi$; that is, one has

$$
\begin{aligned}
\varphi_{x}= & V_{1}=U_{2}=m u u_{y}-2|y|^{m+1} u_{x}^{2}+2(m+2) x u_{x} u_{y}+2 y u_{y}^{2}, \\
\varphi_{y}= & V_{2}=-U_{1}=-m y|y|^{m-1} u u_{x}-(m+2) x y|y|^{m-1} u_{x}^{2} \\
& -4|y|^{m+1} u_{x} u_{y}+(m+2) x u_{y}^{2} .
\end{aligned}
$$

In fact, using the star-shaped hypothesis, one can define

$$
\varphi(P)=\int_{\gamma_{P}} V_{1} d x+V_{2} d y, \quad P \in \Omega^{-},
$$

where $\gamma_{P}$ is the oriented flow line of $D$ from $B=(0,0)$ to a generic point $P$. This potential function vanishes at $B$ and has regularity properties that depend on the regularity of $u$. In particular, $\varphi \in C^{2}\left(\Omega^{-}\right) \cap C^{1}\left(\overline{\Omega^{-}} \backslash\{A, B\}\right) \cap C^{0}(\bar{\Omega})$. One can arrive at $\varphi \in C^{1}\left(\overline{\Omega^{-}}\right)$if one assumes also that $u_{y}, y u_{x}, x u_{x} \in C^{0}\left(\overline{\Omega^{-}}\right)$.

Step 2 (Examine $\left.\varphi_{\mid A B}\right)$. For each $P=(x, 0) \in A B$, one can parametrize $\gamma_{P}(t)=$ $(-t, 0)$ with $t \in[0,-x]$ to find

$$
\varphi(x, 0)=-\int_{0}^{-x} V_{1}(-t, 0) d t
$$

so that

$$
\varphi_{x}(x, 0)=V_{1}(x, 0)=m u u_{y}+2(m+2) x u_{x} u_{y},
$$

but $u(x, 0)=0$ for each $x$, and one concludes that $\varphi$ is constant on $A B$ and vanishes at $B$, so it vanishes identically.

Step 3 (Examine $\left.\varphi_{\mid \Gamma_{1}}\right)$. One defines $v(x)=\varphi(x, y(x))$, where $x \in\left[x_{0}, 0\right]$. Since $u=$ 0 along $\Gamma_{1}$, one has by (4.3) and (4.4) that $v^{\prime}(x)=V_{1}(x, y(x))+V_{2}(x, y(x)) y^{\prime}(x)$ is given by

$$
-\left(2 y+(m+2) x y^{\prime}\right) K u_{x}^{2}+\left(2(m+2) x-4 y K y^{\prime}\right) u_{x} u_{y}+\left(2 y+(m+2) x y^{\prime}\right) u_{y}^{2},
$$


where $K(y)=y|y|^{m-1}$. Now using the fact that $u \equiv 0$ on $\Gamma_{1}$, one has $u_{x}=$ $-u_{y}(d y / d x)$ on $\Gamma_{1}$, which when inserted into (4.5) gives the expression

$$
v^{\prime}(x)=u_{y}^{2}\left(1+y|y|^{m-1}\left(\frac{d y}{d x}\right)^{2}\right)\left(2 y-(m+2) x \frac{d y}{d x}\right) .
$$

Since $\Gamma_{1}$ is subcharacteristic and $D$-starlike, one has $v^{\prime}(x) \leq 0$, and hence $\varphi$ is pointwise decreasing along $\Gamma_{1}$ from $A$ to $C$ with $\max _{\Gamma_{1}} \varphi=\varphi(A)=0$.

Step 4 (Examine $\varphi$ along characteristic segments). For each $P=\left(x_{P}, y_{P}\right) \in \Gamma_{1}$, one considers the characteristic segment of positive slope $[P, Q]_{+}$which connects $P$ to a unique point $Q=(\bar{x}, 0) \in A B$. This curve is given by $(m+2)(x-\bar{x})=$ $-2(-y)^{(m+2) / 2}$ and can be parametrized by

$$
\Gamma(t)=(x(t), y(t))=\left(-\frac{2}{(m+2)}(-t)^{(m+2) / 2}+\bar{x}, t\right), \quad t \in\left[y_{P}, 0\right] .
$$

One considers $w(t)=\varphi(\Gamma(t))$ and finds that

$$
w^{\prime}(t)=\varphi_{y}(\Gamma(t))+(-t)^{m / 2} \varphi_{x}(\Gamma(t))=\left(\partial_{+} \varphi\right)(\Gamma(t)),
$$

where $\partial_{+}=\partial_{y}+(-y)^{m / 2} \partial_{x}$ denotes the directional derivative along characteristics of positive slope. Using the identities (4.2) and (4.3), some calculation shows that

$$
\partial_{+} \varphi=m(-y)^{m / 2} u \partial_{+} u+\left((m+2) x-2(-y)^{(m+2) / 2}\right)\left(\partial_{+} u\right)^{2}
$$

and hence that $w^{\prime}(t)$ does not have a definite sign in general. However, integrating (4.8) along $[P, Q]_{+}$and using the definition of $w$ gives the identity

$$
\varphi(Q)=\varphi(P)+\int_{y_{P}}^{0}\left(\partial_{+} \varphi\right)(\Gamma(t)) d t .
$$

Furthermore, setting $\psi(t)=u(\Gamma(t))$, one has $\psi^{\prime}(t)=\left(\partial_{+} u\right)(\Gamma(t))$, and hence (4.9) and (4.10) give the identity

$\varphi(Q)=\varphi(P)+\int_{y_{P}}^{0} m(-t)^{m / 2} \psi(t) \psi^{\prime}(t)+\left((m+2) \bar{x}-4(-t)^{(m+2) / 2}\right)\left(\psi^{\prime}(t)\right)^{2} d t$.

Finally, integrating by parts on the first term in (4.11) and using the boundary condition, which implies that $\psi\left(y_{P}\right)=0$, yields the identity $\varphi(Q)=\varphi(P)+\int_{y_{P}}^{0} \frac{m^{2}}{4}(-t)^{(m-2) / 2} \psi^{2}(t)+\left((m+2) \bar{x}-4(-t)^{(m+2) / 2}\right)\left(\psi^{\prime}(t)\right)^{2} d t$, 
where the integral can be split into two pieces:

$$
\int_{y_{P}}^{0}(m+2) \bar{x}\left(\psi^{\prime}(t)\right)^{2} d t+\int_{y_{P}}^{0} \frac{m^{2}}{4}(-t)^{(m-2) / 2} \psi^{2}(t)-4(-t)^{(m+2) / 2}\left(\psi^{\prime}(t)\right)^{2} d t .
$$

Both integrals in (4.13) are nonpositive where one uses $\bar{x} \leq 0$ for the first integral and the Hardy-Sobolev inequality given in [18, Lemma 4.3] for the second integral; moreover, the integral in (4.12) is strictly negative unless either $Q=B$ and $P=C$ or $\psi^{\prime} \equiv 0$. Hence $\varphi(Q) \leq \varphi(P)$ for each characteristic segment $[P, Q]_{+}$with strict inequality unless either $Q=B$ and $P=C$ or $\psi^{\prime} \equiv 0$.

Step 5 (Show that $u$ vanishes along characteristic segments). From Steps 2, 3, and 4, one has $0=\varphi(A) \geq \varphi(P) \geq \varphi(Q)=0$ for each pair of points $P \in \Gamma_{1}$ and $Q \in A B$ which are connected by a characteristic segment. Hence $\varphi(P)=0$ for each $P \in \Gamma_{1}$. However, by the observation following formula (4.13), one must have $\varphi(Q)$ strictly negative for each $Q \in[A, B)$ unless $\psi^{\prime} \equiv 0$ along $[P, Q]_{+}$. Since $\varphi(Q)=0$, one has $u \equiv 0$ along $[P, Q]_{+}$for each $Q \in[A, B)$ and hence $u \equiv 0$ on $\overline{\Omega^{-}}$by continuity, which completes the proof.

By using some elliptic and hyperbolic theory, one can modify the argument above to prove the following uniqueness result for mixed-type problems, provided that the starlike and subcharacteristic assumptions made on the various boundary arcs are made in the strict sense. We consider first the so-called Frankl problem, in which the domain $\Omega$ consists of a Goursat domain which is capped off with an elliptic region; that is, the boundary is formed by $\Gamma_{1} \cup B C \cup \sigma$, where $\Gamma_{1}, B C$ are subcharacteristic and characteristic arcs as defined above and $\sigma$ is a piecewise $C^{1}$ simple arc in the elliptic region (where $y>0$ ) which connects $A$ to $B$ (which is again normalized to lie at the origin). Such a domain is called a Frankl domain.

\section{THEOREM 4.2}

Let $\Omega$ be a Frankl domain that is star-shaped with respect to the vector field $D=$ $-(m+2) x \partial_{x}-2 y \partial_{y}$. Assume that $\Gamma_{1}$ is strictly subcharacteristic and that $\Gamma_{1}$ and $\sigma$ are strictly D-starlike; that is, $1+y|y|^{m-1}(d y / d x)^{2}>0$ along $\Gamma_{1}$ and $((m+2) x, 2 y) \cdot v>$ 0 along $\Gamma_{1} \cup \sigma$, where $v$ is the exterior unit normal. Let $u$ be a classical solution of the Frankl problem; $u$ satisfies (4.1) in $\Omega$ with $u=0$ on $\sigma \cup \Gamma_{1}$. Then $u$ is unique.

\section{Proof}

Given that $\Omega$ is $D$-star-shaped, one introduces the dilation potential $\varphi$ as in (4.4), which vanishes in $B=(0,0)$ and is defined on all of $\bar{\Omega}$. As in Theorem 4.1, one analyzes the monotonicity properties of $\varphi$ along various curves. First, using $u=0$ 
along $\Gamma_{1}$ and repeating Step 3 of the proof, one finds (4.6), and hence $\varphi$ is pointwise decreasing along $\Gamma_{1}$ and strictly decreasing along $\Gamma_{1}$ at each point $P$ such that $u_{y}(P) \neq 0$. Next, after choosing a piecewise $C^{1}$-parametrization $(x(t), y(t))$ for $\sigma$ with the orientation which leaves the interior of $\Omega$ on the left, one finds

$$
\varphi_{\mid \sigma}^{\prime}(t)=\left(u_{y}^{2}+y^{m} u_{x}^{2}\right)\left(((m+2) x(t), 2 y(t)) \cdot\left(-y^{\prime}(t), x^{\prime}(t)\right)\right),
$$

where the scalar product is strictly negative (at all but a finite number of points where the normal may not be defined defined). Hence $\varphi$ is decreasing along $\sigma$ (with respect to its orientation) and is strictly decreasing in each point $P$ such that $u_{y}(P) \neq 0$. Finally, repeating the argument of Step 4, one again arrives at formula (4.12), which one needs now only for the characteristic segment $P=C, Q=B$ (and hence $\bar{x}=0$ ). The Hardy-Sobolev inequality again yields $\varphi(B) \leq \varphi(C)$.

\section{CLAIM 1}

One has $\varphi=0=u_{y}$ on $\sigma \cup \Gamma_{1}=0$.

\section{Proof}

One has $\varphi(A) \geq \varphi(C) \geq \varphi(B)=0$ by using the monotonicity along $\Gamma_{1}$ and the comparison along $[C, B]_{+}$. On the other hand, the monotonicity along $\sigma$ gives $\varphi(A) \leq \varphi(B)=0$ and hence $\varphi(A)=0$, which gives the claim for $\varphi$. Since $\varphi$ vanishes along $\sigma \cup \Gamma_{1}$, it is nowhere strictly monotone, and the observations above imply that $u_{y}$ must vanish identically along these curves.

As a result, one has $L u=0$ in $\Omega$ and $u=u_{y}=0$ on $\sigma \cup \Gamma_{1}$, from which hyperbolic theory in $\Omega^{-}$plus maximum principles in subdomains of $\Omega$ give $u \equiv 0$. The argument is by contradiction. Assume that $u \in C^{0}(\bar{\Omega})$ achieves a positive maximum $M=$ $u\left(P_{M}\right)$ for some $P_{M} \in \bar{\Omega}$. (The argument for a negative minimum is analogous.) One has $P_{M} \notin \sigma \cup \Gamma_{1}$.

\section{CLAIM 2}

One has $P_{M} \notin \Omega^{-}$.

\section{Proof}

One decomposes $\Omega^{-}$into two parts. One defines $\Omega_{2}^{-}$as the characteristic triangle $E C B$, where $E=\left(x_{E}, 0\right)$ is the unique point on the $x$-axis connected to $C$ by a characteristic segment $[E, C]_{-}$of negative slope. Since $\Gamma_{1}$ is subcharacteristic, $[E, C]_{-}$ lies entirely in $\overline{\Omega^{-}}$. Then one defines $\Omega_{1}$ as the interior of $\Omega^{-} \backslash \Omega_{2}^{-}$. For each point $\left(x_{0}, y_{0}\right) \in \Omega_{1}^{-}$, consider the backward light cone $\Sigma_{-}\left(x_{0}, y_{0}\right)$ with vertex in $\left(x_{0}, y_{0}\right)$. One has that $y$ is a timelike variable for the strictly hyperbolic operator $L$, where $\Gamma_{1} \cap \Sigma_{-}\left(x_{0}, y_{0}\right)$ is a spacelike curve for $L$ since $\Gamma_{1}$ is subcharacteristic. Hence the 
value of $u$ in $\Omega^{-} \cap \Sigma_{-}\left(x_{0}, y_{0}\right)=\Omega_{1}^{-} \backslash \Sigma_{-}\left(x_{0}, y_{0}\right)$ is uniquely determined by the Cauchy data on $\Gamma_{1} \cap \Sigma_{-}\left(x_{0}, y_{0}\right)$, which vanishes. Hence one has $u=0$ in $\Omega_{1}^{-}$, and hence, by continuity, $u=0$ on $\overline{\Omega_{1}^{-}}$, so that $P_{M} \notin \overline{\Omega_{1}^{-}}$. On $\Omega_{2}^{-}$, one may then apply the Agmon-Nirenberg-Protter maximum principle ([1, Theorem $\left.1^{\prime}\right]$ ) since $L u=0$ and $u=0$ on the characteristic $[E, C]_{-}$which forms part of the boundary. Since $P_{M}$ must also give a positive maximum for $u$ over $\overline{\Omega_{2}^{-}}$, their result applied to $\Omega_{2}^{-}$shows that the maximum cannot occur on $\Omega_{2} \cup B C$. Hence the positive maximum over $\overline{\Omega^{-}}$ must occur along $A B$, and hence one has the claim. (In fact, the maximum must occur along $E B$ since $u$ vanishes on $\overline{\Omega_{1}^{-}}$.)

Hence $P_{M} \in E B \cup \Omega^{+}$, but $P \in E B$ is impossible since $u_{y}\left(P_{M}\right)>0$ in that case (see [1, Theorem $\left.2^{\prime}\right]$ ). This leaves $\Omega^{+}$, which is impossible as well by the Hopf maximum principle applied to $L$ which is strictly and uniformly elliptic on each elliptic subdomain. This completes the proof of Theorem 4.2.

It is clear that an analogous uniqueness theorem can be stated for other boundary value problems, in particular, for $D$-star-shaped Guderley-Morawetz domains in which one removes, from a noncharacteristic domain, a backward light cone with vertex at $Q$ on the interior of the sonic line (where $y=0$ ). Translation invariance in $x$ allows one to place $Q$ at the origin and use the above arguments on both sides of $Q$. We note that Theorem 4.1 includes also the case of the Tricomi problem in which $\Gamma_{1}$ is a characteristic; however, in that special boundary geometry the uniqueness result follows directly from the maximum principle of [1], as does the analog of Theorem 4.2 in the case of the Tricomi problem.

We finish this section with a uniqueness result for the Goursat problem for the semilinear Gellerstedt equation with critical exponent (see Example 3.2). The present result can be thought of as the nonexistence of nontrivial solutions and hence can be compared to [18, Theorem 4.5]. There are two differences here. The present result works at the critical exponent but with a negative constant multiplying the nonlinearity. This second aspect makes the result similar to the nonexistence of nontrivial solutions to $\Delta u+f(u)=0$ with Dirichlet boundary conditions when $s f(s)<0$ for $s \neq 0$. (One multiplies by $u$ and integrates by parts.) In the present case, the corresponding Dirichlet integral is indefinite, and hence such a simple argument does not work here.

\section{THEOREM 4.3}

Let $\Omega^{-}$be a Goursat domain that is star-shaped with respect to the vector field $D=$ $-(m+2) x \partial_{x}-2 y \partial_{y}$. Assume that $\Gamma_{1}$ is subcharacteristic. Let $u$ be a classical solution of the semilinear Goursat problem: $u$ satisfies $y|y|^{m-1} u_{x x}+u_{y y}+C u|u|^{p-2}=0$ in 
$\Omega^{-}$with $u=0$ on $A B \cup \Gamma_{1}$, where $C \leq 0$ and $p=2^{*}(1, m)=2(m+4) / m$. Then $u \equiv 0$ on $\overline{\Omega^{-}}$.

\section{Sketch of the proof}

The argument is that of Theorem 4.1, where one checks that the critical power nonlinearity generates a new potential $\Phi$, which is related to the linear potential $\varphi$ via

$$
\nabla \Phi=\nabla \varphi+(4 y,-2(m+2) x) F(u)
$$

where $F(u)=C|u|^{p} / p$ is the primitive vanishing for $u=0$. In particular, the monotonicity along $\Gamma_{1} \cup A B$, which depends on $\nabla \Phi$, is unchanged since $u=F(u)=0$ there. As for the comparison along characteristic segments $[P, Q]_{+}$for $P \in \Gamma_{1}$, $Q \in A B$, the analog of formulas (4.12) and (4.13) becomes

$$
\begin{aligned}
\Phi(Q)= & \Phi(P)+\int_{y_{P}}^{0}\left(\frac{m^{2}}{4}(-t)^{(m-2) / 2} \psi^{2}(t)-4(-t)^{(m+2) / 2} \psi^{\prime}(t)^{2}\right) d t \\
& +(m+2) \bar{x} \int_{y_{P}}^{0}\left(\psi^{\prime}(t)^{2}-2 F(\psi(t))\right) d t
\end{aligned}
$$

where again $\psi(t)=u(\Gamma(t))$ with $\Gamma(t)$ parameterizing $[P, Q]_{+}$as in (4.7). In fact, using (4.14), the term in the integrand coming from nonlinearity is

$$
-2 F(\psi(t))\left((m+2) x(t)-2 y(t) x^{\prime}(t)\right)=-2(m+2) \bar{x} F(\psi(t)) .
$$

The integrals in (4.15) are nonpositive: the first by the Hardy-Sobolev inequality and the second using $\bar{x} \leq 0$ and the form of $F(\psi)=C|\psi|^{p} / p$ with $C \leq 0$. Hence one has $\Phi(Q) \leq \Phi(P)$ with strict inequality unless $P=C$ and $Q=B$ or $u \equiv 0$ along $[P, Q]_{+}$. As in Theorem 4.1, one concludes that $u \equiv 0$ on all of $\overline{\Omega^{-}}$.

\section{Energy estimates for problems with supercritical growth}

In this section, we exploit the invariance under anisotropic dilations (of the linear part) to the problem of obtaining localized energy estimates for semilinear equations of degenerate hyperbolic type. In particular, we are interested in a priori estimates that give information on the solutions as the time variable tends to a critical instant in which the wave speed tends to zero as a power of the time variable. Beginning with the work of Struwe [33] for radial solutions in $\mathbf{R}^{3}$, such estimates have been obtained for semilinear wave equations at critical and supercritical growth and provide a key step toward proving the global regularity of solutions to the Cauchy problem without small initial energy assumptions. When combined with $\left(L^{p}-L^{q}\right)$-estimates of Strichartz type (see [32]), regularity for critical growth problems has been proven in higher dimensions and for not-necessarily-radial solutions (see [29], [13]). From the point of 
view of mixed-type equations, the estimates we derive give some $L^{p}$-control coming from the hyperbolic side.

We begin by noting the presence of another notion of critical growth for semilinear Gellerstedt equations,

$$
y|y|^{m-1} \Delta_{x} u+\partial_{y}^{2} u+u|u|^{p-2}=0, \quad m>0, p \geq 1,
$$

which for $y \leq 0$ can be thought of as a semilinear wave equation with variable wave speed $|y|^{m}$ which tends to zero as the timelike variable $y$ tends to zero. The exponent

$$
2^{* *}=2^{* *}(N, m)=\frac{2 N(m+2)}{N(m+2)-4}
$$

can be shown to be critical for the total energy functional at time $y$,

$$
E(u ; y)=\int_{\mathbf{R}^{N}}\left(\frac{1}{2}\left(u_{y}^{2}+|y|^{m}\left|\nabla_{x} u\right|^{2}\right)+\frac{1}{p}|u|^{p}\right) d x,
$$

via dimensional analysis (see [30] for the case of wave equations). Indeed, by setting $l$ as a dimension of length for $y$ and $l^{\delta}$ as a dimension of length for each component $x_{j}$ with $j=1, \ldots, N$, one must choose $\delta=(m+2) / 2$ in order to balance the units in the operator $L$. It follows that

$$
\operatorname{dim}(u)=l^{-2 /(p-2)} \quad \text { and } \quad \operatorname{dim}(E(u ; y))=l^{N(m+2) / 2-2-4 /(p-2)}
$$

and hence that the energy is dimensionless for $p=2^{* *}$ and has positive (negative) dimension for $p$ greater (less) than $2^{* *}$. We note that $2^{* *}=2 N^{*} /\left(N^{*}-2\right)$ is the classical Sobolev exponent for $N^{*}=N \delta=N(m+2) / 2$, the homogeneous dimension of the space variables $x \in \mathbf{R}^{N}$, as explained in Section 3. In this section, by critical growth we mean $p=2^{* *}(N, m)>2^{*}(N, m)$, where $2^{*}$ is given by (3.14).

Before giving the results, we establish some notation that is used throughout. For $S, T \leq 0$, we denote by

$$
K_{S}^{T}=\left\{(x, y) \in \mathbf{R}^{N+1}:(m+2)|x|<2(-y)^{(m+2) / 2}, S<y<T\right\}
$$

the truncated backward light cone with vertex at the origin whose boundary is the union of two balls $D(S) \cup D(T)$, where

$$
D(y)=\left\{(x, y) \in \mathbf{R}^{N+1}:(m+2)|x| \leq 2(-y)^{(m+2) / 2}\right\}, \quad y<0,
$$

and the mantle

$$
M_{S}^{T}=\partial K_{S}^{T} \backslash(D(S) \cup D(T)) .
$$

We omit the superscript $T$ in the case $T=0$. We also denote the localized energy on $D(y)$ by 


$$
E(u ; D(y))=\int_{D(y)}\left(\frac{1}{2}\left(u_{y}^{2}+|y|^{m}\left|\nabla_{x} u\right|^{2}\right)+F(u)\right) d x,
$$

where $F(u)$ is always assumed to be nonnegative. The choice of placing the vertex of $K_{S}$ at the origin with respect to the space variables is no restriction due to the translation invariance in $x$.

We begin with a local energy identity.

\section{LEMMA 5.1}

Let $u$ be a classical solution of (5.1) in $K_{S}^{T}$. Then

$$
E(u ; D(S))=E(u ; D(T))+\operatorname{Flux}\left(u ; M_{S}^{T}\right)+\frac{1}{2} \int_{K_{S}^{T}} m|y|^{m-1}\left|\nabla_{x} u\right|^{2} d x d y,
$$

where

$$
\operatorname{Flux}\left(u ; M_{S}^{T}\right)=\int_{M_{S}^{T}}\left(y|y|^{m-1} u_{y} \nabla_{x} u, \frac{1}{2}\left(u_{y}^{2}+|y|^{m}\left|\nabla_{x} u\right|^{2}\right)+F(u)\right) \cdot v d \sigma \geq 0
$$

and where $v$ is the exterior unit normal and $E$ is defined by (5.3).

Proof

One merely takes the $u_{y}$ multiplier identity (3.7) with $K(y)=-|y|^{m}$,

$$
\operatorname{div}\left(y|y|^{m-1} u_{y} \nabla_{x} u, \frac{1}{2}\left(u_{y}^{2}+|y|^{m}\left|\nabla_{x} u\right|^{2}\right)+F(u)\right)=-\frac{m}{2}|y|^{m-1}\left|\nabla_{x} u\right|^{2},
$$

integrates it over $K_{S}^{T}$, and applies the divergence theorem to find (5.4) and the expression (5.5) for Flux $\left(u ; M_{S}^{T}\right)$. It remains only to show that the flux is nonnegative. One has

$$
v=\left(1+|y|^{m}\right)^{-1 / 2}\left(\frac{x}{|x|},|y|^{m / 2}\right),
$$

and hence a short calculation shows that the flux is given by

$$
\operatorname{Flux}\left(u ; M_{S}^{T}\right)=\int_{M_{S}^{T}} \frac{|y|^{m / 2}}{\left(1+|y|^{m}\right)^{1 / 2}}\left(F(u)+\left.\frac{1}{2}|| y\right|^{m / 2} \nabla_{x} u-\left.\frac{x}{|x|} u_{y}\right|^{2}\right) d \sigma,
$$

which is nonnegative if $F(u) \geq 0$.

From Lemma 5.1, since all of the terms in formula (5.4) are nonnegative, one sees that $E(u ; D(T)) \leq E(u ; D(S))$, and so the localized energy decreases as $y<0$ increases and is bounded below by zero, which yields

$$
\lim _{y \rightarrow 0^{-}} E(u ; D(y))=E_{0} \geq 0 .
$$


Moreover, by fixing $S<0$ and letting $T$ tend to zero, one finds that the last two terms in (5.4) are increasing and bounded from above and hence admit finite limits $\operatorname{Flux}\left(M_{S}\right)$ and $G(S)$, respectively, which satisfy the relation

$$
E(u ; D(S))=E_{0}+\operatorname{Flux}\left(M_{S}\right)+G(S) .
$$

It follows that the last two terms in (5.9) admit finite (nonnegative) limits for $S \rightarrow 0^{-}$, which must then be zero. As a result, while there may be local energy concentration along the balls $D(S)$ for $S \rightarrow 0^{-}$since $E_{0}>0$ is possible, there cannot be concentration of the flux along the mantles $M_{S}$ or of the $L^{2}$-norm of $|y|^{(m-1) / 2}\left|\nabla_{x} u\right|$ along the solid backward light cones $K_{S}$. Our main result shows that for supercritical problems, the potential energy $\int_{D(S)}|u|^{p} / p d x$ cannot concentrate along balls (cf. [30, Lemma 6.1]).

\section{THEOREM 5.2}

Let $u$ be a classical solution of $u_{y y}-|y|^{m} \Delta_{x} u+u|u|^{p-2}=0$ on $K_{S}$ with $N \geq 3$, $S<0$, and $p>2^{* *}(N, m)>2$ with $2^{* *}$ given by (5.2). Then there exist constants $C=C(N, m, p)>0$ and $\alpha=\alpha(N, m, p)>1$ such that $-\alpha-1+N(m+2)(p-$ 2) $/ 2 p>0$ and

$$
\begin{gathered}
\int_{D(S)}|u|^{p} d x \leq C\left(|S|^{\alpha-1} E(u ; D(S))+|S|^{-\alpha-1+N(m+2)(p-2) / 2 p} E(u ; D(S))^{2 / p}\right. \\
\left.+\operatorname{Flux}\left(u ; M_{S}\right)+\operatorname{Flux}\left(u ; M_{S}\right)^{2 / p}\right),
\end{gathered}
$$

and hence $\int_{D(S)}|u|^{p} d x \rightarrow 0$ for $S \rightarrow 0^{-}$.

\section{Proof}

For $S<T<0$, one integrates the dilation identity (3.13) over the truncated cone $K_{S}^{T}$ and applies the divergence theorem to find that

$$
-\int_{D(S)} Q d x+\int_{D(T)} Q d x+\int_{M_{S}^{T}}(P, Q) \cdot v d \sigma+\int_{K_{S}^{T}} R d x d y=0
$$

where

$$
\begin{aligned}
Q= & y\left(u_{y}^{2}+|y|^{m}\left|\nabla_{x} u\right|^{2}+\frac{2}{p}|u|^{p}\right)+u_{y}\left((m+2) x \cdot \nabla_{x} u+\gamma u\right), \\
P= & \frac{(m+2)}{2}\left(-u_{y}^{2}+|y|^{m}\left|\nabla_{x} u\right|^{2}+\frac{2}{p}|u|^{p}\right) x \\
& -|y|^{m}\left((m+2) x \cdot \nabla_{x} u+2 y u_{y}+\gamma u\right) \nabla_{x} u, \\
R= & |u|^{p}\left(\gamma-\frac{N(m+2)+2}{p}\right)
\end{aligned}
$$


and where we have denoted by $\gamma=(N(m+2)-2) / 2$ the coefficient of $u$ in the dilation multiplier (3.4). Separating out the potential energy on $D(S)$ from the first integral in (5.11), one finds the identity

$$
\begin{aligned}
2 S \int_{D(S)}|u|^{p} / p d x= & -\int_{D(S)} \widetilde{Q} d x+\int_{D(T)} Q d x \\
& +\int_{M_{S}^{T}}(P, Q) \cdot v d \sigma+\int_{K_{S}^{T}} R d x d y \\
:= & -I+I I+I I I+I V
\end{aligned}
$$

where we have denoted $\widetilde{Q}=Q-2 y|u|^{p} / p$.

In order to arrive at estimate (5.10), the plan is as follows.

(1) Estimate from below the terms to the right in (5.12) (by quantities that are either $o(1)$ for $T \rightarrow 0^{-}$or $o\left(|S|^{\alpha}\right)$ for $S \rightarrow 0^{-}$with $\left.\alpha>1\right)$.

(2) Divide by $S<0$.

(3) Take the limit as $T \rightarrow 0^{-}$.

We begin by noting that $I V \geq 0$ if $p \geq 2^{*}(N, m)$ and hence if $p>2^{* *}(N, m)$. For the integral $-I$, one notes that $\widetilde{Q}=y\left(u_{y}^{2}+|y|^{m}\left|\nabla_{x} u\right|^{2}\right)+u_{y}\left((m+2)\left(x \cdot \nabla_{x} u\right)+\gamma u\right)$ can be rewritten as

$$
\widetilde{Q}=y\left(\left|\widetilde{\nabla}_{x} u\right|^{2}-u_{r}^{2}\right)+y\left(\partial_{+} u\right)^{2}+\gamma u u_{y},
$$

where $\widetilde{\nabla}_{x}=|y|^{m / 2} \nabla_{x}$ is a weighted gradient and

$$
\partial_{r}=\frac{m+2}{2 y}\left(x \cdot \nabla_{x}\right), \quad \partial_{+}=\partial_{y}+\partial_{r}
$$

are suitable directional derivatives, the first being radial and the second being tangential along $M_{S}^{T}$. On $K_{S}^{T}$, where $(m+2)|x| \leq 2|y|^{(m+2) / 2}$, one has $u_{r}^{2} \leq\left|\widetilde{\nabla}_{x} u\right|^{2}$ and hence

$$
-I \geq-\gamma \int_{D(S)} u u_{y} d x .
$$

This integral can be estimated via Young's inequality with $|S|^{\alpha}$ as a parameter and Hölder's inequality to give

$$
\begin{aligned}
\left|\int_{D(S)} u u_{y} d x\right| & \leq 2|S|^{\alpha} E(u ; D(S))+4^{-1}|S|^{-\alpha} \int_{D(S)} u^{2} d x \\
& \leq C\left(|S|^{\alpha} E(u ; D(S))+|S|^{-\alpha+N(m+2)(p-2) / 2 p} E\left(u ;(D(S))^{2 / p}\right),\right.
\end{aligned}
$$

where we have used $|D(S)|=C_{N, m}|S|^{N(m+2) / 2}$. Hence there exists $C_{1}>0$ such that

$$
-I \geq C_{1} S\left(|S|^{\alpha-1} E(u ; D(S))+|S|^{-\alpha-1+N(m+2)(p-2) / 2 p} E(u ; D(S))^{2 / p}\right),
$$


where we need to ensure that $\alpha>1$ can be chosen in such a way as to have $-\alpha+$ $N(m+2)(p-2) / 2 p>1$. Assuming that $p=2^{* *}+\epsilon$ for some $\epsilon>0$, it suffices to pick $\alpha=1+\delta$ with $\delta>0$ such that

$$
2 \delta<N(m+2)-4 \quad \text { and } \quad \delta<\frac{\epsilon(N(m+2)-4)^{2}}{4 N(m+2)+2 \epsilon(N(m+2)-4)} .
$$

To estimate II I from below, we rewrite the integral as

$$
\begin{aligned}
I I I & =\int_{M_{S}^{T}} \frac{|y|^{m / 2}}{\left(1+|y|^{m}\right)^{1 / 2}}\left(2 y\left(\partial_{+} u\right)^{2}+\gamma u \partial_{+} u\right) d \sigma \\
& =\int_{D(S) \backslash D(T)}\left[2 g(x)\left(\partial_{+} u\right)^{2}(x, g(x))+\gamma\left(u \partial_{+} u\right)(x, g(x))\right] d x,
\end{aligned}
$$

where we have used expressions (5.6) and (5.13) for the external normal on $M_{S}^{T}$ and the directional derivative $\partial_{+} u$ and have represented $M_{S}^{T}$ as a graph $y=$ $g(x)=-((m+2)|x| / 2)^{2 /(m+2)}$ over the domain $D(S) \backslash D(T)$. Introducing $v(x)=$ $u(x, g(x))$, one finds that

$$
\partial_{+} u(x, g(x))=u_{y}(x, g(x))+\frac{m+2}{2 g(x)}\left(x \cdot \nabla_{x} u\right)(x, g(x))=\frac{m+2}{2 g(x)}(x \cdot \nabla v)(x),
$$

so that (5.16) becomes

$$
I I I=\int_{D(S) \backslash D(T)}\left(2 g\left(\frac{m+2}{2 g} x \cdot \nabla v\right)^{2}+\gamma \frac{m+2}{2 g} v x \cdot \nabla v\right) d x .
$$

Completing the square in the integrand of (5.17) and integrating by parts yields

$$
\begin{aligned}
I I I= & \int_{D(S) \backslash D(T)} \frac{1}{2 g(x)}[(m+2) x \cdot \nabla v+\gamma v]^{2} d x \\
& -\frac{\gamma}{4}(m+2) \int_{\partial(D(S) \backslash D(T))} \frac{1}{g(x)} v^{2} x \cdot v d \sigma,
\end{aligned}
$$

where we recall $\gamma=(N(m+2)-2) / 2$. Hence one has

$$
\begin{aligned}
I I I= & \int_{D(S) \backslash D(T)} \frac{1}{2 g(x)}\left[2 g(x) \partial_{+} u(x, g(x))+\gamma u(x, g(x))\right]^{2} d x \\
& +\frac{\gamma}{2}|S|^{m / 2} \int_{\partial D(S)} u^{2} d \sigma-\frac{\gamma}{2}|T|^{m / 2} \int_{\partial D(T)} u^{2} d \sigma \\
\geq & A-B:=\int_{D(S) \backslash D(T)} \frac{1}{2 y}\left[2 y \partial_{+} u+\gamma u\right]^{2} d x-\frac{\gamma}{2}|T|^{m / 2} \int_{\partial D(T)} u^{2} d \sigma,
\end{aligned}
$$


where in the last formula $u$ and its derivatives are calculated in $(x, g(x))$ and $y=$ $g(x)<0$.

The expression $A$ can be estimated from below by flux integrals, where we rewrite (5.7) as

$$
\operatorname{Flux}\left(u ; M_{S}^{T}\right)=\int_{D(S) \backslash D(T)}\left(\frac{|u|^{p}}{p}+\frac{1}{2}|\widetilde{\nabla} u|^{2}\right) d x
$$

by parameterizing $M_{S}^{T}$ as above and defining

$$
\widetilde{\nabla} u=|y|^{m / 2} \nabla_{x} u-\frac{x}{|x|} u_{y} .
$$

The integrand of $A$ in (5.18) satisfies

$$
\frac{1}{2 y}\left[2 y \partial_{+} u+\gamma u\right]^{2}=\frac{1}{2 y}\left[2 y\left(\frac{-x}{|x|} \cdot \widetilde{\nabla} u\right)+\gamma u\right]^{2} \geq 4 y|\widetilde{\nabla} u|^{2}+\frac{1}{y} \gamma^{2} u^{2},
$$

from which it follows that

$$
\begin{aligned}
A & \geq 8 \operatorname{SFlux}\left(u ; M_{S}^{T}\right)+\gamma^{2} \int_{D(S) \backslash D(T)} \frac{u^{2}}{y} d x \\
& \geq 8 \operatorname{SFlux}\left(u ; M_{S}^{T}\right)+\gamma^{2} S \int_{D(S)} \frac{u^{2}}{y^{2}} d x .
\end{aligned}
$$

Then, by Hölder's inequality, one has

$$
\begin{aligned}
\int_{D(S)} \frac{u^{2}}{y^{2}} d x & \leq C\left(\int_{D(S)}|x|^{-2 p /(p-2)} d x\right)^{(p-2) / p}\left(\int_{D(S)}|u|^{p} d x\right)^{2 / p} \\
& \leq C_{2} \operatorname{Flux}\left(u ; M_{S}\right)^{2 / p}
\end{aligned}
$$

with $C_{2}>0$ finite for $p>2^{* *}$. Combining (5.18), (5.19), and (5.20) with the monotonicity of the flux in $T$ yields $C_{3}>0$ such that

$$
I I I \geq C_{3} S\left(\operatorname{Flux}\left(u ; M_{S}\right)+\operatorname{Flux}\left(u ; M_{S}\right)^{2 / p}\right)-\frac{\gamma}{2}|T|^{m / 2} \int_{\partial D(T)} u^{2} d \sigma .
$$

Now, combining (5.12) with (5.14), (5.21), and the fact that $I V \geq 0$ yields

$$
\begin{aligned}
\int_{D(S)}|u|^{p} d x \leq & C\left(|S|^{\alpha-1} E(u ; D(S))+|S|^{-\alpha-1+N(m+2)(p-2) / 2 p} E(u ; D(S))^{2 / p}\right. \\
& \left.+\operatorname{Flux}\left(u ; M_{S}\right)+\operatorname{Flux}\left(u ; M_{S}\right)^{2 / p}\right) \\
+ & \frac{p}{2 S}\left(I I-\frac{\gamma}{2}|T|^{m / 2} \int_{\partial D(T)} u^{2} d \sigma\right)
\end{aligned}
$$

which gives the desired inequality (5.10) if the last two terms tend to zero with $T$. 


\section{CLAIM 1}

One has $I I=\int_{D(T)} Q d x \rightarrow 0$ for $T \rightarrow 0^{-}$.

\section{Proof}

Indeed, one splits $Q=2 y e+u_{y}\left((m+2) x \cdot \nabla_{x} u+\gamma u\right)$, where $e=\left(u_{y}^{2}+\right.$ $\left.|y|^{m}\left|\nabla_{x} u\right|^{2}\right) / 2+|u|^{p} / p$ is the energy density associated to (5.3). Applying Young's inequality with $|T|$ as a parameter gives

$$
\left.\left|u_{y}\left((m+2) x \cdot \nabla_{x} u+\gamma u\right)\right| \leq\left.|T|\left(u_{y}^{2}+4^{-1}|T|^{-2} \mid(m+2) x \cdot \nabla_{x} u+\gamma u\right)\right|^{2}\right),
$$

where $(m+2)|x| \leq 2|T|^{(m+2) / 2}$ on $D(T)$, and hence a short calculation shows that

$$
\left|Q_{\mid D(T)}\right| \leq|T|\left(8 e+2^{-1}|T|^{-2} \gamma^{2} u^{2}\right)
$$

Thus, by Hölder's inequality, one has

$$
|I I| \leq C|T|\left(E(u ; D(T))+|T|^{-2+N(m+2)(p-2) / 2 p} E(u ; D(T))^{2 / p}\right),
$$

where the exponent $-2+N(m+2)(p-2) / 2 p$ is nonnegative for $p \geq p^{* *}(N, m)$, which completes the claim by (5.8).

\section{CLAIM 2}

One has $|T|^{m / 2} \int_{\partial D(T)} u^{2} d \sigma \rightarrow 0$ for $T \rightarrow 0^{-}$.

Proof

It is enough to show that there exist $\alpha, \beta, q>0$ such that

$$
|T|^{m / 2} \int_{\partial D(T)} u^{2} d x \leq C\left(|T|^{\alpha} E(u ; D(T))+|T|^{\beta} E(u ; D(T))^{q}\right) .
$$

This follows from Hölder's inequality after exploiting the inequality

$$
\int_{\partial B_{R}} u^{2} d \sigma \leq \frac{N+1}{R} \int_{B_{R}} u^{2} d x+R \int_{B_{R}}\left|\nabla_{x} u\right|^{2} d x
$$

which holds for regular functions on the ball $B_{R}$ of radius $R$ in $\mathbf{R}^{N}$, which we apply on $D(T)=B_{R}$ with $(m+2) R=2|T|^{(m+2) / 2}$. One finds the estimate (5.22) with $q=2 / p, \alpha=1$, and $\beta=-1+N(m+2)(p-2) / 2 p$, where $\beta>0$ if $p>$ $2 N(m+2) /[N(m+2)-2]$ and hence if $p \geq p^{* *}(N, m)$. This completes the proof of the claim.

Hence Theorem 5.2 is proved. 
Remarks. The restriction $N \geq 3$ is used primarily in estimate (5.20) for II I but also ensures that the left-hand member in (5.15) is positive. The argument leading to (5.14) exploits supercritical growth, which can be adjusted to the case of critical growth using a different lower estimate for $\tilde{Q}$ in the first integral of (5.12) (analogous to the estimate used in [30]). The needed sign condition holds provided $N \geq 4$, and hence Theorem 5.2 holds for critical growth if $N \geq 4$.

\section{Concluding remarks}

In this section, we collect some comments on our results concerning the underlying geometric structure and applications of equations of the form (1.1). We begin by noting that mixed-type equations of the form (1.1) arise in a wide variety of problems with a very particular structure. The oldest and most well-studied occurs in the plane in the context of modeling transonic flow, as originated in the work of Frank1 [7]. One considers a planar, irrotational, stationary, compressible, and isentropic flow whose velocity field $(U, V)$ then admits a velocity potential $\varphi$ and stream function $\psi$ such that $\nabla \varphi=(U, V)$ and $\rho\left(\varphi_{x}, \varphi_{y}\right)=\left(\psi_{y},-\psi_{x}\right)$. The equation for the stream function (or for perturbations in the velocity potential) can be linearized by a suitable hodograph transformation resulting in the equation

$$
K(s) \tilde{\psi}_{\theta \theta}+\tilde{\psi}_{s s}=0,
$$

where $\tilde{\psi}$ is the rescaled stream function obtained by dividing by the product of critical speed and pressure, $s$ is a logarithmic rescaling of the flow speed, and $\theta$ is the flow angle (cf. [3, Section 3]). In the case of transonic flow, one has $K(s) \sim(1+\gamma) s$ for $s \rightarrow 0$, where $\gamma>1$ is the exponent in the adiabatic pressure-density relation, and hence (6.1) is a mixed-type equation whose type-change function is linear in $s$ and hence well approximated by the Tricomi equation $K(s)=s$ for nearly sonic speeds $s=0$ after a trivial change of variables. Moreover, the Frankl problem (or the Tricomi problem in a limiting case) is the correct boundary value problem to solve in the hodograph plane for a transonic flow in a symmetric nozzle. The subcharacteristic arc $\Gamma_{1}$ corresponds to the jet boundary at the exit, which is a streamline for the flow, and the characteristic $B C$, on which no data is placed, corresponds to localizing the problem near the exit of the nozzle since, in general, downstream shocks form, and hence the hodograph method breaks down (cf. [9, Chapter 14]). Hence our uniqueness result in Theorem 4.2 gives qualitative information on this transonic flow problem without assuming that the jet boundary is monotone near the exit. Moreover, uniqueness theorems for such problems in the hodograph plane are the basis for showing nonexistence of continuous flows via perturbation methods, as was pioneered by Morawetz [20]. In addition, we note that the conservation laws we have obtained for the Tricomi equation resemble those obtained by Ovsiannikov (see [25, Section 5]) for the von Kármán 
system

$$
U_{y}=V_{x}, \quad V_{y}=-U U_{x},
$$

which gives a transonic approximation to the flow field $(U, V)$ in the physical plane under the hypothesis of a nearly horizontal flow (cf. [3, Section 4]). Ovsiannikov has shown that the system (6.2) has a four-dimensional group of symmetries corresponding to translations in $V$, an anisotropic dilation in $(U, V)$, and something like an inversion in $(U, V)$, plus the trivial irrotationalilty in the space variables $(x, y)$. Hence our result also suggests that after returning from the hodograph plane one should still have a four-dimensional group of symmetries, even if the flow is not assumed to be nearly horizontal.

Mixed-type equations of the form (1.1) also arise in geometric problems. In particular, in the paper of Lin [16] it is shown that the problem of locally isometrically embedding a two-dimensional surface whose Gauss curvature $K$ changes sign into $\mathbf{R}^{3}$ gives rise to nonlinear equations of mixed type. More precisely, given the first fundamental form $I=E d u_{1}^{2}+2 F d u_{1} d u_{2}+G d u_{2}^{2}$ in local coordinates near a point $P$ on the surface, one seeks three functions $(x, y, z)\left(u_{1}, u_{2}\right)$ such that $I=d x^{2}+d y^{2}+d z^{2}$ in a neighborhood of $P$. If the curvature vanishes at $P$, then each unknown component, for example, $z$, satisfies a certain second-order Monge-Ampere equation. In the case where $K$ changes sign cleanly at $P(K(P)=0, \nabla K(P) \neq 0)$, after an inessential normalization and a suitable change of independent and dependent variables $(u, z) \mapsto(U, Z)$, one has the equation

$$
U_{2} Z_{U_{1} U_{1}}+Z_{U_{2} U_{2}}+\epsilon f\left(\epsilon, U, D Z, D^{2} Z\right)=0,
$$

where $\epsilon$ is a small stretching parameter and $f$ is smooth in its arguments. Thus one has a small nonlinear perturbation of the linear Tricomi equation. If one relaxes the cleanness of the sign change in $K$, one arrives at a more general mixed-type equation, where other positive powers of $U_{2}$ are of particular interest as they can be used to describe the order of degeneration and result in a nonlinear Gellerstedt equation. Our experience suggests that the dilation multiplier should give rise to the needed estimates on the linearizations of (6.3) in order to extend Lin's embedding result to surfaces whose curvature changes sign in a nonclean way. Similar considerations in higher dimension yield equations or systems of equations whose local forms are of type (1.1).

The linear operator in equation (1.1) is associated to a singular geometry on $\mathbf{R}^{N+1}$ via the metric $g$, which in global coordinates $(x, y)$ on $\mathbf{R}^{N+1}$ is given by the matrix

$$
\left[g_{i j}\right]=\left[\begin{array}{cc}
K(y)^{-1} I_{N \times N} & 0 \\
0 & 1
\end{array}\right],
$$

which gives, for $K$ smooth and satisfying (1.2) and (1.3), a smooth Riemannian/Lorenzian metric on the upper/lower half-spaces which becomes singular on 
the hyperplane $y=0$. If one computes the associated Laplace-Beltrami operator/D'Alembertian in their respective half-planes, one arrives at the geometrically natural operator

$$
\operatorname{div}_{g} \circ \nabla_{g}=K(y) \Delta_{x}+\partial_{y}^{2}-\frac{N K^{\prime}(y)}{2 K(y)} \partial_{y} .
$$

Hence the linear operator in (1.1) is the principal and everywhere smooth part of this geometric operator. Mixed signature metrics have received some attention in the context of quantum cosmology, beginning with the Hawking-Hartle no-boundary hypothesis, which, if true, implies that space-time evolved from a Riemannian past into a Lorentzian present, as well as models for tunnelling effects in quantum gravity (cf. $[15$, Chapters 3, 5]).

Finally, we note that, as happens for the Laplace and wave equations, the symmetry groups of Theorem 2.5 are strongly related to the conformal transformations with respect to the underlying geometric structure given above. In particular, after neglecting the trivial part of the symmetry group coming from the linearity and homogeneity, that is, the part generated by $\left\{v^{u}, v^{\beta}\right\}$, the rest corresponds to a group of conformal transformations on the base space $\mathbf{R}^{N+1}$ endowed with the singular metric $g$ defined by (6.4). More precisely, away from the metric singularity in $y=0$, one can check that the system of partial differential equations which defines the conformal Killing fields (the infinitesimal generators of a one-parameter group of conformal transformations) is equivalent to the subsystem depending only on $\xi$ and $\eta$ in the system that determines the symmetry group via formula (2.21). This subsystem appears in formulas (A.10) - (A.12) of the appendix. In dimension $N+1 \geq 3$, the proof of Theorem 2.5 shows that this part of the system is finite-dimensional and spanned by the translations, dilations, and inversions above. Moreover, by knowing explicitly these conformal transformations, it is clear that they can be continued across $y=0$ in a smooth way to form an $(N+1+N(N-1) / 2)$-dimensional group of conformal transformations even though the underlying metric is singular along $y=0$. In dimension $N+1=2$, the group is infinite-dimensional, but only a finite-dimensional subgroup corresponds to a symmetry group for (2.19). The connection between the full geometric operator (6.5) and such conformal groups will be discussed in a forthcoming note [26].

\section{A. Appendix}

In this appendix, we sketch the proof of Theorem 2.5. In order to apply the infinitesimal criteria of Lemma 2.4, we need to verify that the equation satisfies the needed nondegeneracy conditions. One thinks of equation (2.19) as defining a subvariety

$$
\mathscr{S}_{\mathscr{F}}=\left\{\left(x, y ; u^{(2)}\right): \mathscr{F}\left(x, y ; u^{(2)}\right)=0\right\} \subset \mathbf{R}^{N+1} \times \mathscr{U}^{(2)},
$$


where for $m \in \mathbf{N}, \mathscr{F}: \mathbf{R}^{N+1} \times \mathscr{U}^{(2)} \rightarrow \mathbf{R}$ is a smooth map defined by

$$
\mathscr{F}\left(x, y ; u^{(2)}\right)=y^{m} \sum_{j=1}^{N} u_{x_{j} x_{j}}+u_{y y}
$$

and $\mathbf{R}^{N+1} \times \mathscr{U}^{(2)}$ is the 2-jet space of independent and dependent variables together with the second-order derivatives of the dependent variable up to order two, which for smooth solutions can be given coordinates

$$
\left\{\left(x, y ; u^{(2)}\right)=\left(x, y ; u_{x_{i}}, u_{x_{i} x_{j}}, u_{x_{i} y}, u_{y y}\right): 1 \leq i \leq j \leq N\right\},
$$

which makes $\mathbf{R}^{N+1} \times \mathscr{U}^{(2)} \simeq \mathbf{R}^{N+1} \times \mathbf{R} \times \mathbf{R}^{N+1} \times \mathbf{R}^{\left(N^{2}+4 N+2\right) / 2}$. One says that the differential equation (2.19) is nondegenerate if it satisfies the following two conditions (cf. [24, Definitions 2.30, 2.70]).

(i) The equation is of maximal rank in the sense that the Jacobian matrix $J_{\mathscr{F}}$ of (A.1) satisfies

$$
\operatorname{rank}\left(J_{\mathscr{F}}\right)=1 .
$$

(ii) The equation is locally solvable in the sense that for each fixed $P_{0}=$ $\left(x_{0}, y_{0} ; u_{0}^{(2)}\right) \in \mathbf{R}^{N+1} \times \mathscr{U}^{(2)}$, there exists a smooth solution $u=f(x, y)$ to the equation in a neighborhood of $P_{0}$ with these prescribed values; that is,

$$
f^{(2)}\left(x_{0}, y_{0}\right)=u_{0}^{(2)} \text {. }
$$

For equation (2.19), it follows easily that the equation is of maximal rank, since $\mathscr{F}_{u_{y y}}=1 \neq 0$, and is locally solvable since a suitable polynomial solution $f$ is easily constructed as a Taylor polynomial for the given data.

We may now apply Lemma 2.4 to construct the bases of the various infinitesimal symmetries of the theorem. The claim that the set of generators in parts (a) and (b) forms a Lie algebra with respect to the commutator bracket is a general fact for nondegenerate equations (cf. [24, Corollary 2.40, Proposition 4.16]). To construct the bases, one can use the general formula of Olver for the coefficients of the prolonged vector fields (cf. [24, Theorem 2.36]), and so the rest of the proof amounts to a sequence of lengthy calculations, which we briefly summarize.

\section{Proof of Theorem 2.5(a)}

The second prolongation of a vector field $v$ of the form (2.20) is given by

$$
\operatorname{pr}^{(2)} v=v+\sum_{j=1}^{N} \varphi^{x_{j}} \frac{\partial}{\partial u_{x_{j}}}+\varphi^{y} \frac{\partial}{\partial u_{y}}+\sum_{j, k=1}^{N} \varphi^{x_{j} x_{k}} \frac{\partial}{\partial u_{x_{j} x_{k}}}+\sum_{j=1}^{N} \varphi^{x_{j} y} \frac{\partial}{\partial u_{x_{j} y}}+\varphi^{y y} \frac{\partial}{\partial u_{y y}}
$$


where the coefficients $\varphi^{x_{j}}, \varphi^{y}$, and so on, are calculated via a general formula and the expressions $u_{x_{j}}, u_{y}$, and so on, represent the coordinates in the higher-order jet spaces. In particular, one finds that

$$
\begin{aligned}
\varphi^{x_{j} x_{j}}= & \varphi_{x_{j} x_{j}}+2 \varphi_{x_{j} u} u_{x_{j}}+\varphi_{u u} u_{x_{j}}^{2}+\varphi_{u} u_{x_{j} x_{j}} \\
& -\sum_{i=1}^{N}\left[u_{x_{i}}\left(\xi_{x_{j} x_{j}}^{i}+2 \xi_{x_{j} u}^{i} u_{x_{j}}+\xi_{u u}^{i} u_{x_{j}}^{2}+\xi_{u}^{i} u_{x_{j} x_{j}}\right)\right] \\
& -u_{y}\left[\eta_{x_{j} x_{j}}+2 \eta_{x_{j} u} u_{x_{j}}+\eta_{u u} u_{x_{j}}^{2}+\eta_{u} u_{x_{j} x_{j}}\right] \\
& -2 \sum_{i=1}^{N} u_{x_{i} x_{j}}\left(\xi_{x_{j}}^{i}+\xi_{u}^{i} u_{x_{j}}\right)-2 u_{x_{j} y}\left(\eta_{x_{j}}+\eta_{u} u_{x_{j}}\right),
\end{aligned}
$$

and one uses a similar form for $\varphi^{y y}$, which are the only coefficients needed since equation (2.19) depends only on $y, u_{x_{j} x_{j}}, u_{y y}$. Applying (2.21) yields a polynomial in $1, u, D u, D^{2} u$ with coefficients that depend on $\xi, \eta, \varphi$, and their partial derivatives up to order two. Equating this polynomial with zero, collecting monomials, substituting $-y^{m} \Delta_{x} u$ for $u_{y y}$ whenever it appears, and eliminating redundant or trivial equations yields the following system for $(\xi, \eta, \varphi)$ as functions of $(x, y, u)$ :

$$
\begin{aligned}
L \varphi=y^{m} \sum_{j=1}^{N} \varphi_{x_{j} x_{j}}+\varphi_{y y} & =0, \\
L \xi^{i}=y^{m} \sum_{j=1}^{N} \xi_{x_{j} x_{j}}^{i}+\xi_{y y}^{i} & =2 y^{m} \varphi_{x_{i} u}, \quad i=1, \ldots, N, \\
L \eta=y^{m} \sum_{j=1}^{N} \eta_{x_{j} x_{j}}+\eta_{y y} & =2 \varphi_{y u}, \\
\xi_{u}^{i} & =0, \\
y^{m} \eta_{u} & =0, \\
\varphi_{u u}-2 \eta_{y u} & =0, \\
2 y^{m} \xi_{x_{i}}^{i}-2 y^{m} \eta_{y}-m y^{m-1} \eta & =0, \quad i=1, \ldots, N, \\
y^{m}\left(\xi_{x_{j}}^{i}+\xi_{x_{i}}^{j}\right) & =0, \quad 1 \leq i<j \leq N, \\
y^{m} \eta_{x_{i}}+\xi_{y}^{i} & =0, \quad i=1, \ldots, N .
\end{aligned}
$$

Using (A.7) -(A.9) plus regularity, one finds that $\xi^{i}, \eta$ are independent of $u$ and that $\varphi=\alpha(x, y) u+\beta(x, y)$ is linear in $u$. In particular, since $u \equiv 0$ and $u \equiv 1$ are solutions, one sees that $\alpha, \beta$ must be solutions of equation (2.19) and that (A.4) - (A.6) 
can be rewritten as

$$
\begin{aligned}
L \alpha & =L \beta=0, \\
L \xi^{i} & =2 y^{m} \alpha_{x_{i}}, \\
L \eta & =2 \alpha_{y},
\end{aligned}
$$

where $\beta$ is free to be any solution of (2.19). The rest of the calculation splits according to the spatial dimension.

Case 1: Dimension $N \geq 2$. In this case, one can show that the subsystem (A.10)(A.12) has a finite-dimensional solution space given by

$$
\begin{aligned}
\xi^{i}(x, y)= & a_{0}^{i}+a_{1}^{1} x_{i}+\sum_{\substack{j=1 \\
j \neq i}}^{N} a_{j}^{i} x_{j}+a_{1 i}^{1}\left(-\frac{2}{(m+2)^{2}} y^{m+2}-\frac{1}{2}|x|^{2}\right) \\
& +x_{i} \sum_{j=1}^{N} a_{1 j}^{1} x_{j}, \\
\eta(x, y)= & \frac{2}{m+2} a_{1}^{1} y+\sum_{j=1}^{N} \frac{2}{m+2} a_{1 j}^{1} x_{j} y,
\end{aligned}
$$

where $\left\{a_{0}^{i}, a_{1}^{1}, a_{j}^{i}, a_{1 i}^{1}\right\}$ are real coefficients. In fact, one can show that most higherorder derivatives vanish (such as $\eta_{x_{i} x_{k}}, \xi_{x_{k} y}^{i}$ with $i \neq k$ and $\xi_{x_{j} x_{k}}^{i}$ with $i \neq j \neq k \neq i$ in the case $N \geq 3$ ) and that $\eta_{x_{1} x_{1}}=\ldots=\eta_{x_{N} x_{N}}$, which is then used to show that $\xi^{i}, \eta$ are quadratic polynomials in $x$ with $y$ dependent coefficients. Inserting these polynomials into the subsystem and using the explicit smooth solutions to the equation

$$
2 y g^{\prime}(y)+m g(y)=2 A y, \quad A \in \mathbf{R},
$$

one arrives at (A.13) and (A.14). In short, the argument is similar to that used in the classical computation for the (constant coefficient) wave operator, and the principal difficulty here is to allow for the $y$-dependence everywhere.

To finish the claim of part (a) in this case, one merely inserts (A.13) and (A.14) into $\left(\right.$ A. $\left.4^{\prime}\right)-\left(\right.$ A. $\left.6^{\prime}\right)$ and calculates to find that

$$
\alpha=\alpha(x)=\alpha_{0}+\sum_{i=1}^{N} \frac{2-N(m+2)}{2(m+2)} a_{1 i}^{1} x_{i},
$$

where $\alpha_{0}$ is a new constant. Combining (A.13), (A.14), and (A.15) shows that the general vector field $v$ is a linear combination of the vector fields $v^{u}, v^{\beta}, v_{i}^{T}, v_{i j}^{R}, v_{i}^{I}$ and the field

$$
\tilde{v}^{D}=v^{D}+\frac{N(m+2)-2}{2} v^{u},
$$


which gives a basis equivalent to that claimed in (2.29). The preferred basis (2.29) could be called a conservation law basis in light of parts (b) and (c) of the theorem.

Case 2: $N=1$. Here, the subsystem (A.10) - (A.12) reduces to a pair of equations

$$
2 y^{m} \xi_{x}-2 y^{m} \eta_{y}-m y^{m-1} \eta=0=y^{m} \eta_{x}+\xi_{y}
$$

for two unknown functions in two variables and has an infinite-dimensional solution space. However, coupling (A.17) with (A.4') - (A.6 $)$ yields a finite-dimensional space of $v$ 's as these relations constrain most higher-order derivatives of $\xi, \eta, \alpha$ to vanish. In particular, one finds that $\alpha_{y}=\alpha_{x x}=0$ and hence that $\eta_{y y}=\eta_{x x}=\xi_{x y}=\xi_{x x x}=$ 0 . Using these reductions, one easily arrives at the basis $\left\{v^{u}, v^{\beta}, v^{T}, \tilde{v}^{D}, \tilde{v}^{I}\right\}$. This completes the proof of Theorem 2.5(a).

\section{Proof of Theorem 2.5(b)}

Here it is enough to note that having selected a vector field that generates a symmetry (from part (a)), one has the following formulas for the coefficients of the first prolongation $\operatorname{pr}^{(1)} v=v+\sum \varphi^{x_{j}} \partial_{x_{j}}+\varphi^{y} \partial_{y}$ :

$$
\varphi^{x_{j}}=\alpha_{x_{j}} u+\beta_{x_{j}}+\alpha u_{x_{j}}-\sum_{i=1}^{N} u_{x_{i}} \xi_{x_{j}}^{i}-u_{y} \eta_{x_{j}},
$$

and the formula is similar for $\varphi^{y}$. Applying formula (2.22), one finds a polynomial in $(1, u, D u)$ which must vanish. One finds that $\alpha$ and $\beta$ must be constant and hence that $a_{i 1}^{1}=0$ and $\alpha_{0}=a_{1}^{1}(2-N(m+2)) /(2(m+2))$. Thus no inversions are allowed, and the dilation $\tilde{v}^{D}$ of (A.16) must be modified to $v^{D}$. This gives the basis of generators, as claimed.

\section{Proof of Theorem 2.5(c)}

The fact that the set of infinitesimal divergence symmetries forms a vector space is obvious from the definition. Moreover, since every variational symmetry is a divergence symmetry (by taking $B=\left(B_{1}, \ldots, B_{N}, B_{N+1}\right)=0$ ), it remains only to check that the inversion symmetries (with $v=v_{k}^{I}$ ) and the trivial symmetries (with $v=v^{\beta}$, where $L \beta=0$ ) are divergence symmetries while the trivial symmetry (with $v=v^{u}$ ) is not. For these three generators, formula (2.23) yields a polynomial in 1, $u, D u$ whose vanishing cannot happen for $v^{u}$, while for $v^{\beta}$ with $L \beta=0$ one can choose $B=\left(y^{m} u \nabla_{x} \beta, u \beta_{y}\right)$ and for $v_{k}^{I}$ one can choose

$$
B_{j}=\delta_{j k} \frac{2-N(m+2)}{4(m+2)} y^{m} u^{2}, \quad j=1, \ldots, N+1 .
$$


Acknowledgments. The authors thank both Cathleen Morawetz for numerous stimulating discussions and the Courant Institute for its hospitality during the period in which this work was begun. The authors also thank two anonymous referees for their helpful comments concerning a prior version of this work.

\section{References}

[1] S. AGMON, L. NIRENBERG, and M. H. PROTTER, A maximum principle for a class of hyperbolic equations and applications to equations of mixed elliptic-hyperbolic type, Comm. Pure Appl. Math. 6 (1953), 455-470. MR 0058835

[2] J. BARROS-NETO and I. M. GELFAND, Fundamental solutions for the Tricomi operator, Duke Math. J. 98 (1999), 465 -483. MR 1695798

[3] L. BERS, Mathematical Aspects of Subsonic and Transonic Gas Dynamics, Surveys Appl. Math. 3, Wiley, New York, 1958. MR 0096477

[4] H. BRÉZIS and L. NIRENBERG, Positive solutions of nonlinear elliptic equations involving critical Sobolev exponents, Comm. Pure Appl. Math. 36 (1983), 437 - 477. MR 0709644

[5] D. ChristodoulOU and S. KLAINERMAN, The Global Nonlinear Stability of the Minkowski Space, Princeton Math. Ser. 41, Princeton Univ. Press, Princeton, 1993. MR 1316662

[6] B. FRANCHI and E. LANCONELLI, Hölder regularity theorem for a class of linear nonuniformly elliptic operators with measurable coefficients, Ann. Scuola Norm. Sup. Pisa Cl. Sci. (4) 10 (1983), 523 - 541. MR 0753153

[7] F. I. FRANKL, On the problems of Chaplygin for mixed sub-and supersonic flows (in Russian), Izvestia Akad. Nauk. SSSR 9 (1945), 121 - 143. MR 0015981

[8] K. O. FRIEDRICHS, Symmetric positive linear differential equations, Comm. Pure Appl. Math. 11 (1958), 333 -418. MR 0100718

[9] P. R. GARABEDIAN, Partial Differential Equations, 2nd ed., Chelsea, New York, 1986. MR 0943117

[10] N. GAROFALO and E. LANCONELLI, Existence and nonexistence results for semilinear equations on the Heisenberg group, Indiana Univ. Math. J. 41 (1992), 71 -98. MR 1160903

[11] S. GELLERSTEDT, Sur un problème aux limites pour une équation linéaire aux dérivées partielles du second ordre de type mixtes, Ph.D. dissertation, Uppsala University, Uppsala, Sweden, 1935.

[12] P. GERMAIN and R. BADER, Sur quelques problèmes relatifs à l'équation de type mixte de Tricomi, O.N.E.R.A. Publ. 1952 (1952), no. 54. MR 0052665

[13] M. G. GRILLAKIS, Regularity of the wave equation with a critical nonlinearity, Comm. Pure Appl. Math. 45 (1992), 749-774. MR 1162370

[14] V. V. GRUSHIN [GRUŠIN], Singularities of solutions of a certain class of pseudodifferential and degenerating elliptic equations (in Russian), Uspekhi Mat. Nauk 26, no. 1, (1971), 221 - 222. MR 0296482

[15] S. HAWKING and R. PENROSE, The Nature of Space and Time, Isaac Newton Inst. Ser. Lectures, Princeton Univ. Press, Princeton, 1996. MR 1372945 
[16] C. S. LIN, The local isometric embedding in $\mathbf{R}^{3}$ of two-dimensional Riemannian manifolds with Gaussian curvature changing sign cleanly, Comm. Pure Appl. Math. 39 (1986), 867 - 887. MR 0859276

[17] D. LUPO and K. R. PAYNE, Spectral bounds for Tricomi problems and application to semilinear existence and existence with uniqueness results, J. Differential Equations 184 (2002), 139 - 162. MR 1929150

[18] Critical exponents for semilinear equations of mixed elliptic-hyperbolic and degenerate types, Comm. Pure Appl. Math. 56 (2003), 403 -424. MR 1941814

[19] C. S. MORAWETZ, Note on a maximum principle and a uniqueness theorem for an elliptic-hyperbolic equation, Proc. Roy. Soc. London Ser. A 236 (1956), 141 - 144. MR 0079712

[20] - On the non-existence of continuous transonic flows past profiles, I, II, Comm. Pure Appl. Math. 9 (1956), 45 -68; Comm. Pure Appl. Math. 10 (1957), 107 - 131. MR 0078130; MR 0088253

[21] - The decay of solutions of the exterior initial-boundary value problem for the wave equation, Comm. Pure Appl. Math. 14 (1961), 561 -568. MR 0132908

[22] - Variations on conservation laws for the wave equation, Bull. Amer. Math. Soc. (N.S.) 37 (2000), 141 - 154. MR 1751947

[23] E. NOETHER, Invarianten beliebiger Differentialausdrücke, Nachr. Ges. d. Wiss. zu Göttingen (Math.-phys. K1.) (1918), $37-44$; also in Gesammelte Abhandlungen, Springer, Berlin, 1983, 240-257. MR 0703862

[24] P. J. OLVER, Applications of Lie Groups to Differential Equations, 2nd ed., Grad. Texts in Math. 107, Springer, New York, 1993. MR 1240056

[25] L. V. OVSIANNIKov, Group Analysis of Differential Equations, Academic Press, New York, 1982. MR 0668703

[26] K. R. PAYNE, Singular metrics and associated conformal groups underlying differential operators of mixed and degenerate types, preprint, 2004, to appear in Ann. Mat. Pura Appl.

[27] S. I. POHOŽAEV, On the eigenfunctions of the equation $\Delta u+\lambda f(u)=0$, Soviet Math. Dokl. 6 (1965), 1408 - 1411. MR 0192184

[28] M. H. PROTTER, Uniqueness theorems for the Tricomi problem, J. Rational Mech. Anal. 2 (1953), 107 - 114. MR 0052663

[29] J. SHATAH and M. STRUWE, Regularity results for nonlinear wave equations, Ann. of Math. (2) 138 (1993), 503 - 518. MR 1247991

[30] - Geometric Wave Equations, Courant Lect. Notes Math. 2, Amer. Math. Soc., Providence, 1998. MR 1674843

[31] W. A. STRAUSS, Nonlinear Wave Equations, CBMS Regional Conf. Ser. in Math. 73, Amer. Math. Soc., Providence, 1989. MR 1032250

[32] R. S. STRICHARTZ, Restrictions of Fourier tranforms to quadratic surfaces and decay of solutions of wave equations, Duke Math. J. 44 (1977), 705-714. MR 0512086

[33] M. STRUWE, Globally regular solutions to the $u^{5}$ Klein-Gordon equation, Ann. Scuola Norm. Sup. Pisa Cl. Sci. (4) 15 (1988), 495 -513. MR 1015805

[34] F. G. TRICOMI, Sulle equazioni lineari alle derivate parziali di $2^{0}$ ordine, di tipo misto, Atti Acad. Naz. Lincei Mem. Cl. Fis. Mat. Nat. (5) 14 (1923), 133 - 247. 
Lupo

Dipartimento di Matematica "F. Brioschi," Politecnico di Milano, Piazza Leonardo da Vinci 32, 20133 Milano, Italy; danlup@mate.polimi.it

Payne

Dipartimento di Matematica “F. Enriques," Università di Milano, Via Saldini 50, 20133 Milano, Italy; payne@mat.unimi.it 